\title{
Untersuchungen über die Abhängigkeit der Gefäß- und Sklerenchymbildung von äußeren Faktoren nebst einigen Bemerkungen über die angebliche Heterorhizie bei Dikotylen.
}

\author{
Von Paul Flaskämper.
}

(Mit 21 Abbildungen im Text.)

Mit Hypertrophie bezeichnete Virchow in der pathologischen Anatomie des Menschen das Größerwerden eines Organs infolge starken Gebrauchs; das bekannteste Beispiel hierfür ist die alltägliche Erscheinung, daß die Muskeln des Menschen durch intensive Inanspruchnahme an Stärke zunehmen, histologisch gesprochen, daß „der Querschnitt des Muskelprimitivbündels wächst, indem sich zwischen den alten neue, quergestreifte Primitivfibrillen ausbilden"1).

Von E. Küster ${ }^{2}$ ) wurden ähnliche Begriffe in die Botanik eingeführt. Küster unterscheidet zwischen Aktivitätshypertrophie und Aktivitätshyperplasie, indem bei jener verstärkte Inanspruchnahme eine Vergrößerung der Zellen, bei dieser eine Vermehrung derselben hervorrufen soll. Es waren besonders zwei Gewebe, bei denen man eine derartige Vermehrung oder Verstärkung ihrer Elemente vermutete: das Leitungsgewebe und das mechanische Gewebe. Besonders daß eine mechanische Inanspruchnahme die Pflanze zu einer vermehrten Produktion von Sklerenchym oder Kollenchym oder zu einer stärkeren Ausbildung der Wandverdickung ihrer mechanisch wirksamen Elemente zwingen würde, hielt man nach Analogie mit den oben erwähnten Verhältnissen beim tierischen Muskel für sehr wahrscheinlich. Hegler glaubte nun in der Tat eine derartige selbstregulatorische Anpassung durch mechanische Beanspruchung und zwar auf Zug gefunden zu haben. Seine Untersuchungen sind von Pfeffer in einer vorläufigen Mitteilung in den Sitzungsberichten der sächsischen Gesellschaft der Wissenschaften zu Leipzig ${ }^{3}$ ) mitgeteilt worden. Hegler behauptete bei verschiedenen Pflanzen, Keimpflanzen von Helianthus annuus, Blattstielen von Helleborus und anderen eine Zunahme der Tragfähigkeit gefunden zu haben,

1) Hertwig, O., Allgemeine Biologie, Jena 1906, pag. 439.

2) Küster, E., Pathologische Pflanzenanatomie, 1903, pag. 65.

3) Berichte der Kgl. Sächs. Gesellsch. d. Wissensch. zu Leipzig, mathem.physik. Klasse 1891, pag. 638. 
die bedingt war durch eine Zunahme der Zahl oder der Wandverdickungen der mechanisch wirksamen Zellen, also des Kollenchyms oder des Sklerenchyms; im Blattstiel von Helleborus niger glaubte er sogar eine Neubildung von dickwandigen Sklerenchym aus dünnwandigen Phloemelementen konstatiert zu haben.

Man mußte also aus den Versuchen Hegler's schließen, daß die Pflanze in ähnlicher Weise wie das Tier auf mechanische Inanspruchnahme reagiere. Diese Angaben, die auch in die 2. Auflage von Pfeffer's Pflanzenphysiologie aufgenommen worden sind ${ }^{1}$ ), wurden später durch eine gründliche Arbeit von Ball2) widerlegt. Doch vor Ball hatten schon andere sich mit dieser Frage beschäftigt, über deren Untersuchungen ich kurz berichten will.

Im Jahre 1902 hatte Vöchting ${ }^{3}$ ) mit Brassica oleracea f. gongylodes, Beta vulgaris und Helianthus, die am Blühen verhindert wurden und deshalb eine starke Zunahme ihres vegetativen Wachstums zeigten, Versuche gemacht. Er hatte sie auf Druck beansprucht durch Belastung bis zu $20 \mathrm{~kg}$. Er erhielt jedoch nur negative Resultate. Im Jahre 1903 machte Wiedersheim ${ }^{4}$ ) Versuche an Trauerbäumen. Er belastete die herabhängenden Zweige dieser Varietäten mit Gewichten und verglich sie mit unbelasteten Zweigen. Er erhielt bei den Trauervarietäten von Fagus silvatica, Sorbus aucuparia und Fraxinus excelsior keine positiven Resultate, nur bei Corylus Avellana erhielt er eine Hyperplasie der Bastelemente im Sinne Küsters. Dieses eine Resultat kann jedoch bei allen übrigen negativen nicht ins Gewicht fallen, da individuelle Schwankungen und andere Einflüsse mit im Spiel gewesen sein können. Ball, dessen Arbeit oben erwähnt wurde, experimentierte sowohl mit denselben Pflanzen wie Hegler, als auch mit einigen anderen. Er kommt nun zu dem Ergebnis, daß die Pflanze sich der Beanspruchung auf Zug nicht anpaßt durch Erhöhung ihres Tragvermögens, daß also von einer Verstärkung oder Vermehrung des Kollen-

1) Pfeffer, Pflanzenphysiologie, II, 1904, pag. 148. Im XII. Kapitel, pag. 425, das offenbar später bearbeitet ist, nachdem die Resultate Ball's bekannt geworden waren, widerruft er diese Angabe jedoch.

2) Ball, Oscar Melville, Der Einfluß von Zug auf die Ausbildung von Festigungsgewebe. Pringsheim's Jahrb. f. wiss. Bot., Bd. XXXIX, pag. 305.

3) Vöchting, Zur experimentellen Anatomie. Nachr. d. Kgl. Gesellsch. d. Wissensch. zu Göttingen, mathem.-physik. Klasse, 1902.

4) Wiedersheim, Walther, Über den Einfluß der Belastung auf die Ausbildung von Holz- und Bastkörper bei Trauerbäumen. Jahrb. f. wissensch. Bot., Bd. XXXVIII, pag. 41. 
Untersuchungen über die Abhängigkeit der Gefäß- u. Sklerenchymbildung usw. 183

chyms oder Sklerenchyms geschweige von einer Neubildung desselben keine Rede sein kann.

Ähnliche Untersuchungen stellte ${ }^{\circ} K^{2} l l e r^{1}$ ) an mit Fruchtstielen. Er verstärkte das Eigengewicht von Früchten durch Anhängen sehr beträchtlicher Gewichte, ohne eine Umbildung der Gewebe zu erhalten. Indem ich die Arbeit von Wildt, auf die ich weiter unten zu sprechen kommen werde, zunächst übergehe, erwähne ich noch kurz die von Hibbard ${ }^{2}$ ). Er untersuchte den Einfluß von Druck und Zug auf die Sprosse verschiedener Pflanzen, erhielt jedoch negative oder nur schwach positive Resultate: Er sagt selbst: „The increase was not great". $\mathrm{Er}$ hat nun auch zum ersten Male mit Wurzeln experimentiert und erhielt dabei sehr schwache positive Resultate. Seine wenigen Ergebnisse, die für eine Anpassung der Pflanze an mechanische Inanspruchnahme zu sprechen scheinen, sind aber ebensowenig entscheidend wie das oben erwähnte Ergebnis Wiedersheim's bei Corylus Avellana var. pendula. Die Annahme individueller Variationen reicht völlig zur Erklärung aus. Ehe ich nun zur Besprechung der beiden Arbeiten übergehe, die für den Ausgang der vorliegenden Untersuchung von Bedeutung sind, nämlich einer Arbeit von Wildt und von Vöchting, möchte ich einige allgemeine Bemerkungen machen. Selbst wenn man sich auf den vitalistischen Standpunkt stellt, daß die Pflanze auf eine Änderung ihrer Umgebung innerhalb gewisser Grenzen in zweckmäßiger Weise reagiert, ist es unwahrscheinlich, daß sie durch eine Beanspruchung auf Zug gezwungen wird, ihr mechanisches Gewebe zu verstärken. In der normalen Pflanze nämlich ist eine so große Menge mechanisch wirksamer Zellen vorhanden, daß dieselbe einen bedeutenden Zug oder Druck aushalten kann, ohne ihr mechanisches Gewebe verstärken zu müssen. Keller (l. c.) z. B. konnte ohne Nachteil für die Pflanze das Gewicht der Früchte von Smilacine um das 390 fache, von Aconitum um das 1600 fache, von Reseda um das 1800 fache erhöhen. Ich selbst habe bei meinen Versuchen derartige Verhältnisse gefunden, über die ich unten berichten werde. Bei Helianthus hat Vöchting ${ }^{3}$ ) die Zug- und Druckfestigkeit gemessen. Er fand, daß ein Stamm der Sonnenrose mindestens $115 \mathrm{~kg}$ tragen kann, ohne zu zerknicken, während er in

1) Keller, Heinrich, Über den Einfluß der Belastung und Lage auf die Ausbildung des Gewebes in Fruchtstielen. Inaug.-Diss., Kiel 1904.

2) Hibbard, The influence of tension on the formation of mechanical tissue in plants. Botanical Gazette, Vol. XLIII, pag. 361.

3) Vöchting, Zur experimentellen Anatomie und Pathologie des Pflanzenkörpers. Tübingen 1908, pag. 274. 
der Natur ein relativ geringes Gewicht zu tragen hat. Er erklärt diese ungeheure mechanische Leistungsfähigkeit nicht für überflüssig, sondern zur Erreichung der nötigen Biegungsfestigkeit für erforderlich. Jedenfalls geht aus diesen Angaben hervor, daß es selbst vom vitalistischen Standpunkte aus gar nicht zu erwarten ist, daß die Pflanze auf eine mechanische Inanspruchnahme durch eine Verstärkung des mechanischen Gewebes antwortet. Andererseits ist es wieder schwer vorstellbar, daß eine derartige starke Veränderung der Lebensbedingungen der Pflanze, wie es der Zug z. B. ist, ohne Wirkung sein soll. Es sind auch schon verschiedene derartige Wirkungen konstatiert worden. So ist z. B. ein Einfluß auf das Wachstum und die Richtung der Zellwände von $\mathrm{Kny}^{1}$ ) festgestellt worden. Er sagt: „Däs Wachstum wird, soweit nicht andere Kräfte entgegenwirken, im Sinne des Zuges und senkrecht zur Richtung des Druckes gefördert. Bei Zellteilungen suchen sich die Scheidewände in die Richtung des Druckes und senkrecht zur Richtung des Zuges zu stellen“" ). Eine Hemmung der kambialen Tätigkeit und ein Kleinerbleiben der Zellen infolge von Druck konnte Prein ${ }^{3}$ ) feststellen. Ich komme auf diese Arbeit im Laufe meiner Untersuchungen noch einmal zu sprechen. Auch auf das Längenwachstum ist ein Einfluß des Zuges festgestellt worden. Durch Untersuchungen von Baranetzky4), $\mathrm{S} \operatorname{cholz}^{5}$ ) und $\mathrm{Hegler}^{6}$ ) ist nachgewiesen worden, daß der Zug zunächst hemmend auf das Längenwachstum wirkt. Nach einer anfänglichen Hemmung tritt dann nach den Untersuchungen der oben genannten Forscher eine Beschleunigung des Wachstums ein. Doch dies nur nebenbei. Wichtiger ist für uns eine Hemmung der Gewebeausbildung, die sich in einigen Fällen nachweisen läßt, wovon ich jedoch weiter unten sprechen werde.

1) Kny, Über den Einfluß von Zug und Druck auf die Richtung der Scheidewände in sich teilenden Pflanzenzellen. Jahrb. f. wiss. Bot., Bd. XXXVII, pag. 55.

2) l. c. pag. 96 .

3) Prein, Rudolf, Über den Einfluß mechanischer Hemmungen auf die histologische Entwicklung der Wurzeln. Diss., Bonn 1908.

4) Baranetzky, J., Die tägliche Periodizität im Längenwachstum der Stengel. Mém. de l'acad. imp. des sciences de St. Petersbourg, VII. Série, 1879, pag. 20 (zitiert nach der folgenden Arbeit von Scholz).

5) Scholz, Max, Über den Einfluß von Dehnung auf das Längenwachstum der Pflanzen. Cohn's Beiträge zur Biologie der Pflanzen, IV, Heft III, pag. 323.

6) Hegler, Rob., Über den Einfluß des machanischen Zuges auf das Wachstum der Pflanze. Cohn's Beiträge zur Biologie der Pflanzen, VI, Heft III, pag. 383. 
Die negativen Ergebnisse der Versuche, die Pflanze durch Zug oder Druck zu einer Verstärkung ihres mechanischen Apparates zu bringen, machen es auch äußert unwahrscheinlich, daß die mechanischen Gewebe phylogenetisch durch äußere Einflüsse mechanischer Art, wie Wind, Regen usw. entstanden sind, wie Nägeli ${ }^{1}$ ) wollte. Es erhebt sich aber nun die Frage, von welchen Faktoren die Ausbildung dieser Gewebe abhängt. $\mathrm{Da} \beta$ allein die Vererbung maßgebend ist, ist nicht anzunehmen, wenn man die beträchtlichen individuellen Schwankungen bedenkt, die das mechanische Gewebe in den verschiedenen Individuen ein und derselben Pflanzenart zeigt. Es existieren nun in der Tat in der Literatur Angaben, die beweisen, daß äußere Faktoren einen maßgebenden Einfluß auf die Ausbildung dieser Gewebe ausüben. Alle diese Angaben lassen letzten Endes mehr oder weniger auf die Abhängigkeit von Ernährungsverhältnissen schließen. Es ist z. B. bekannt, daß die Wasserformen amphibischer Pflanzen weniger Gefäße und verholzte Elemente aufweisen als die entsprechenden Landformen ${ }^{2}$ ). Keller ${ }^{3}$ ) weist darauf hin, daß die Fruchtstiele von Aquilegia und Podophyllum in trockenen Sommern mehr mechanisches Gewebe ausbilden, als in feuchten Sommern. Die gründlichsten Untersuchungen in dieser Beziehung sind von den französischen Anatomen gemacht worden. Laurent ${ }^{4}$ ) z. B. gibt an, daß hoher Wassergehalt der Pflanze (z. B. bei Wasserkulturen) die Verholzung herabsetzt, Kulturen äuf Glukose und anderen Kohlehydraten aber sie erhöhen. Ferner gibt er an, daß etiolierte Pflanzen sich auszeichnen ,par la réduction de l'appareil de soutien, c'est à dire des tissus lignifiés"; als Grund gibt er Mangel an Kohlehydraten an, der durch die Verhinderung der Assimilation entstanden ist. Einen Fall, der besonders deutlich die Abhängigkeit der Sklerenchymbildung von der Transpiration zeigt, eine Beziehung, auf die ich auch im weiteren Verlauf meiner Arbeit noch mehrmals hinweisen werde, will ich noch kurz erwähnen. Goebel ${ }^{5}$ ) fand bei Exemplaren von Festuca ovina var. glauca, die er in feuchter

1) v. Naegeli, C., Mechanisch-physiologische Theorie der Abstammungslehre. München u. Leipzig 1884, pag. 146.

2) Goebel, Pflanzenbiologische Schilderungen, II, Marburg 1893, pag. 232.

3) Keller, Heinrich, Über den Einfluß der Belastung und Lage auf die Ausbildung des Gewebes in Fruchtstielen. Diss., Kiel 1904.

4) Laurent, J., Les facteurs de la structure chez les végétaux. Revue gén. de Bot., Tome XIX, pag. 129.

5) Goebel, Einleitung in die experimentelle Morphologie der Pflanzen. Leipzig u. Berlin 1908, pag. 28. 
Luft unter einer Glasglocke, also bei stark gehemmter Transpiration, kultivierte, daß die Wandverdickung der Sklerenchymfasern in den Blättern stark rückgebildet, teilweise auch die Anlegung der letzteren unterblieben war. Auch das mechanische Gewebe in den Gallen gehört hierher ${ }^{1}$ ). Denn der entweder durch das eierlegende Insekt oder wie in den meisten Fällen die aus den Eiern sich entwickelnde Larve ausgeübte Reiz, der wahrscheinlich auf der Wirkung von Enzymen beruht, beeinflußt die Ernährungsverhältnisse in bestimmter Weise, wenn auch hier die Beziehungen nicht so klar sind, wie in anderen Fällen. Es kann nach allen diesen Angaben keinem Zweifel unterliegen, daß die Ausbildung der mechanischen Gewebe abhängig ist, abgesehen von einer Vererbung, von Ernährungsverhältnissen, nicht aber von mechanischer Inanspruchnahme. Scheinbar im Widerspruch mit dieser Behauptung stehen nun die Ergebnisse zweier Untersuchungen, die Vöchting und Wildt angestellt haben und von denen meine Untersuchungen ausgehen. Vöchting ${ }^{2}$ ) kultivierte Kürbisse am Boden liegend und freihängend und fand, daß die Fruchtstiele der letzteren Früchte mehr Sklerenchym enthielten, als die der ersteren. Er schließt daraus, daß das Gewicht eines zur Pflanze gehörigen Organes die Reaktion auszulösen imstande sei, die ein angehängtes Gewicht als Fremdkörper nicht auslösen könne. Die andere Arbeit von $\mathrm{Wildt}^{3}$ ) beschäftigt sich mit Wurzeln, die eine Anpassung an den Zug zeigen sollen. Die letztere besteht zwar nicht in einer Vermehrung oder Verstärkung der mechanisch wirksamen Bestandteile, sondern in einer anderen Anordnung derselben, die er als zugfestere Konstruktion betrachtet. Er geht dabei aus von einer Unterscheidung der Wurzeln in Ernährungs- und Befestigungswurzeln, die von Tschirch ${ }^{4}$ ) eingeführt worden ist. Da diese Heterorhizie, wie Tschirch diese Erscheinung nennt, die Basis der Wild t'schen Arbeit bildet, muß ich zunächst etwas ausführlicher von letzterer sprechen und meine eigenen Untersuchungen in dieser Frage anführen. Dann werde ich die Ergebnisse der.Wildt'schen Arbeit

1) Vergl. besonders E. Küster, Beiträge zur Kenntnis der Gallenanatomie. Flora 1900, Bd. 87, pag. 117.

2) Vöchting, Untersuchungen zur experimentellen Anatomie und Pathologie des Pflanzenkörpers. Tübingen 1908, pag. 287.

3) Wildt, Willi, Über die experimentelle Erzeugung von Festigkeitselementen in Wurzeln und deren Ausbildung in verschiedenen Nährböden. Diss., Bonn 1906.

4) Tschirch, A., Über die Heterorhizie bei Dikotylen. Flora 1905, Bd. 94, pag. 69 . 
näher beschreiben und diskutieren. Wir werden sehen, daß die Vöchting'schen und Wildt'schen Resultate dem oben ausgesprochenen Satze betreffs der Abhängigkeit des mechanischen Gewebes von den Faktoren der Ernährung nicht widersprechen.

Tschirch, der den Begriff der Heterorhizie einführt, glaubte nämlich bei einer ganzen Reihe dikotyler Pflanzen eine Differenzierung ihres Wurzelsystems in zwei ganz verschiedenen Funktionen, nämlich der der Ernährung und der der Befestigung, angepaßte Typen gefunden zu haben. Seine eigenen Worte sind: „Diese Heterorhizie ist, wie unsere Untersuchungen lehren, eine bei den Dikotylen weit - man kann fast sagen allgemein verbreitete Erscheinung. Der Fall ist relativ selten, wo eine und dieselbe Wurzel sowohl der Ernährung, wie der Befestigung dient $\left.{ }^{1}\right)^{*}$. Sehen wir einmal $\mathrm{zu}$, worin sich die beiden Typen unterscheiden sollen und prüfen wir zunächst, ob diese anatomischen Differenzen überhaupt auf eine derartige Arbeitsteilung schließen lassen. Den Unterschied gibt Tschirch folgendermaßen an: Die Befestigungswurzeln zeigen entweder „einen zentralen Holzkörper ohne Libriform oder einen zentralen Libriformzylinder (meist mit eingestreuten Gefäßen) oder einen zentralen Holzkörper mit Libriformstreifen. Mark pflegt den Befestigungswurzeln zu fehlen. Die Ernährungswurzeln dagegen zeigen in der Regel keinerlei mechanische Elemente und besitzen stets ein mehr oder weniger großes Mark“"2). Sieht man von dem Vorhandensein oder Fehlen der mechanischen Elemente ab, die ja Tschirch selbst nur bei einigen der von ihm untersuchten Pflanzen gefunden hat, so besteht der wichtigste Unterschied zwischen den beiden Typen nach obigem Zitat von Tschirch und nach seinen Abbildungen in dem Fehlen oder Vorhandensein von Mark und in der geringeren Entwicklung des Holzkörpers bei den Ernährungswurzeln und der größeren Entwicklung desselben bei den Befestigungswurzeln. Es ist aber klar, daß eine Wurzel, gleichgültig, ob sie in der Mitte des Zentralzylinders Mark enthält oder nicht, dieselbe mechanische Leistungsfähigkeit haben kann, wenn nur die Zahl und Stärke der Gefäße bei Abwesenheit anderer mechanisch wirksamer Bestandteile dieselbe ist; denn ,die Zugfestigkeit hängt einzig und allein von der Größe des Querschnittes der widerstandsfähigen Elemente $a b^{\text {“3). }}$ ). Bei Valeriana officinalis haben nun beide Typen ein Mark; der Unter-

1) Tschirch, l. c. pag. 71 .

2) Tschirch, l. c. pag. 78 .

3) Schwendener, S., Das mechanische Prinzip im Bau der Monokotylen. Leipzig 1874, pag. 116. 
schied besteht also lediglich in der Entwicklung des Holzkörpers. Da aber die Gefäße doch nicht nur zur Befestigung, sondern vor allem zur Stoffleitung, d. h. zur Ernährung dienen, ist nicht recht einzusehen, inwiefern die Wurzeln mit starkem Holzkörper nur der Befestigung dienen sollen, nicht auch der Ernährung, ja sogar noch mehr als die Wurzeln mit geringerem Holżkörper, die Tschirch Ernährungswurzeln nennt. Auf das Fehlen oder Vorhandensein mechanischer Elemente, sowie andere Einzelheiten im anatomischen Bau dieser beiden Typen komme ich unten näher zu sprechen. Doch ich will die weiteren Anhaltspunkte, die sich aus der bloßen Betrachtung der anatomischen Bilder für die ungeeignete Bezeichnung der beiden Typen ergeben, nicht weiter verfolgen. Wir wollen uns vielmehr die Frage vorlegen, ob die Behauptung Tschirch's richtig ist, daß Ernährungs- und Befestigungswurzeln zwei so scharf getrennte Typen sind wie z. B. die Haft- und Nährwurzeln mancher Epiphyten, daß sie also für gewöhnlich durch keinen Übergang verknüpft sind. Meine Untersuchungen führten zu dem Ergebnis, daß diese Ansicht irrig ist. Schon bei Betrachtung der Tschirch'schen Abbildungen, namentlich der von Valeriana officinalis, kann man sich der Vermutung nicht erwehren, daß die Befestigungswurzeln nichts weiter sind als ältere Stadien der Ernährungswurzeln. Diese Vermutung wurde auch im Verlaufe meiner Untersuchungen bestätigt. Ich konnte beobachten, daß Ernährungs- und Befestigungswurzeln nicht streng geschiedene Typen sind, sondern durch zahlreiche Übergänge kontinuierlich verbunden sind; andererseits konnte ich sehen, daß Wurzeln, die an der Basis das typische Bild einer Befestigungswurzel zeigten, nach der Spitze zu allmählich in Ernährungswurzeln übergingen. $\mathrm{Zu}$ ähnlichen Resultaten gelangte v. Alten ${ }^{1}$ ) in einer kürzlich erschienenen Arbeit. Er faßt seine Untersuchungen über diese Frage zusammen in den Worten: „Wir sehen also, daß bei krautigen Pflanzen die älteren Wurzeln sukzessive durch jüngere ersetzt werden. Beide Wurzelsorten sind in ihrem anatomischen Bau verschieden, aber dieser Unterschied wird nicht durch eine verschiedene physiologische Funktion, sondern lediglich durch das ungleiche Alter bedingt. Auch die jüngeren Wurzeln (die Ernährungswurzeln Tschirch's) gehen im Alter in einen Bau über, wie ihn zu derselben Zeit schon die älteren „Befestigungswurzeln“ zeigen“2). Ich gehe jetzt dazu über, die von

1) v. Alten, Hermann, Wurzelstudien. Botan. Ztg. 1909, I. Abteilung, Heft $10 / 11$.

2) v. Alten, Hermann, l. c. pag. 183 . 
mir auf Heterorhizie untersuchten Fälle zu beschreiben, soweit sie besonders für unsere Zwecke Interesse bieten.

Bei Valeriana officinalis, das Tschirch als erstes Beispiel mit besonderer Ausführlichkeit behandelt, fand ich folgende Verhältnisse. Die Anfang November aus dem System des Münchener botanischen Gartens entnommenen Wurzeln zeigten nur den von Tschirch als Ernährungswurzel bezeichneten Typus. Schon diese Tatsache mußte mir die Bedeutung der sogenannten Befestigungswurzeln zweifelhaft machen, da die Pflanze ohne den Besitz derartiger Wurzeln der nötigen Festigkeit im Boden entbehrt hätte. Ich vermutete den Grund für das Fehlen der Befestigungswurzeln darin, daß die Ernährungswurzeln als die jüngeren im Laufe des Sommers angelegt werden und in diesem Zustande überwintern, um im nächsten Jahre zu „Befestigungswurzeln“ weiter zu wachsen, die dann im Laufe der Vegetationsperiode absterben. Im Winter konnten also nur die von Tschirch als Ernährungswurzeln bezeichneten jüngeren Stadien angetroffen werden. Die aus der pharmazeutischen Sammlung unseres Instituts entnommenen Exemplare zeigten dieselben Verhältnisse wie jene aus dem botanischen Garten, d. h. es ließen sich nur Ernährungswurzeln finden. Es hängt dies damit zusammen, daß die Droge, Radix Valerianae, „besteht aus höchstens $5 \mathrm{~cm}$ langen Rhizomen, welche die Endknospe und meist einige kürzere Zweige tragen“1). Die Ausläufer oder Rhizome, die im Laufe der Vegetationsperiode gebildet werden und deren Endknospen im nächsten Jahre die Blüten tragen, können natürlich nur junge Wurzeln haben. Auch wird die Droge im September eingesammelt ${ }^{2}$ ), zu einer Zeit also, wo die alten Wurzeln schon abgestorben sind. Ich untersuchte dann im Sommer des folgenden Jahres im Freien gesammeltes Material. Hieran mußte ich nun, wenn die oben ausgesprochene Vermutung richtig war, beide von Tschirch aufgestellte Typen finden. In der Tat gelang mir das auch. Ich konnte aber auch bei Betrachtung einer größeren Anzahl von Wurzeln sämtliche Übergänge je nach dem Alter der Wurzel finden. Noch wichtiger aber ist die Tatsache, daß eine Wurzel, deren Querschnittsbild an der Basis einer Befestigungswurzel glich, weiter nach der Spitze zu in eine Ernährungswurzel überging. Ich konnte diese Beobachtung sehr oft machen. Ich will zur besseren Anschauung einige Zahlen hierfür geben. Der Übergang von dem einen Typus in

1) Berg u. Schmidt, Atlas der offizinellen Pflanzen, Bd. I, Leipzig 1893, pag. 26.

2) Hager, H., Kommentar zum Arzneibuch für das Deutsche Reich, Bd. II, Berlin 1896, pag. 492 . 
den anderen war vollendet einmal in einer Entfernung von $5 \mathrm{~cm}$ von der Basis, einmal in einer Entfernung von $6 \mathrm{~cm}$, einmal in einer Entfernung von $9 \mathrm{~cm}$. Je älter die Wurzel ist, desto länger ist natürlich die Strecke von der Basis aus gerechnet, während welcher sie den Typus der Befestigungswurzel beibehält. Von Alten nennt nun in seiner oben zitierten Arbeit „das durch ungleiches Alter bedingte verschiedene Verhalten"1) Dimorphismus. Er beruft sich dabei auf Freidenfelt ${ }^{2}$ ). Vom Dimorphismus scheidet er streng die Heterorhizie, unter der er die verschiedene Ausbildung von Haupt- und Nebenwurzeln versteht. Heterorhizie in diesem Sinne habe Tschirch überhaupt nicht beschrieben nach der Meinung von Altens, sondern nur Dimorphismus. Beide Begriffe halte ich für überflüssig, denn die Erscheinung, daß eine Wurzel in verschiedenen Entwicklungsstadien ein verschiedenes anatomisches Bild zeigt, bedarf ebensowenig eines besonderen Begriffes wie der Unterschied zwischen den Haupt- und Nebenwurzeln verschiedener Ordnung. Die Nebenwurzeln sind immer anatomisch einfacher gestaltete Formen, charakteristisch durch ihre Reduktion der Rinde, der Zahl der Gefäße und der Gefäßstrahlen und des Marks im Zentralzylinder. Eine von mir untersuchte Nebenwurzel von Valeriana officinalis hatte nur acht bis neun Zellschichten in der Primärrinde außer der Epidermis, während die Zahl derselben bei den Hauptwurzeln zwischen 18 und 20 schwankt.

Die übrigen von mir untersuchten Pflanzen haben nun zu demselben Resultat geführt, nämlich, daß sich Übergänge finden zwischen den beiden Typen, sowohl an einer und derselben Wurzel in verschiedener Entfernung von der Basis als auch an der Basis verschiedener Wurzeln. Eine Differenzierung in zwei verschiedenen Funktionen angepaßte Formen ist also nicht vorhanden. Ich konnte dies bestätigt finden bei folgenden von Tschirch angeführten Pflanzen: Ranunculus acer, Artemisia vulgaris, Aconitum Napellus, Aconitum japonicum.

Ausführlicher möchte ich noch sprechen über Aconitum japonicum. Mir schien der Unterschied zwischen „Ernährungs- und Befestigungswurzel" hier besonders interessant, weil bei den Befestigungswurzeln Sklereiden in Mark und Rinde vorkommen sollten, nicht aber in den Ernährungswurzeln. Die Mitte Oktober oder etwas später untersuchten Pflanzen, die aus dem Münchener botanischen Garten stammten, zeigten zunächst nur Ernährungswurzeln im Sinne Tschirch's, d. h. meist

1) v. Alten, Hermann, l. c. pag. 184.

2) Freidenfelt, T., Studien über die Wurzeln krautiger Pflanzen. Flora 1902, Bd. 91, pag. 115 . 
hexarche Wurzeln ohne merkliches sekundäres Dickenwachstum und mit gewöhnlichen parenchymatischen Zellen im Mark und in der primären Rinde, also ohne irgendwelche Sklereiden. Da alle an der Pflanze befindlichen noch lebenden Wurzeln dasselbe Bild zeigten, vermutete ich, daß die Verhältnisse hier ähnlich wie bei Valeriana officinalis lagen, d. h. daß die Wurzeln in diesem Zustand überwintern, um im nächsten Jahre durch Ausbildung von Sklereiden sich in „Befestigungswurzeln“ umzuwandeln. Da nun an den mir zur Verfügung stehenden Pflanzen sich noch die im Vorjahre gebildete Knolle mit den Wurzeln, wenn auch in abgestorbenem Zustand befand, so mußte sich an diesen Wurzeln, wenn meine Vermutung richtig war, der Tschirch'sche Typus der Befestigungswurzel nachweisen lassen. In der Tat konnte ich an ihnen, obgleich sie nur noch in kurzen Stücken vorhanden und schon ganz braun und teilweise verfault waren, das Vorhandensein von Sklereiden nachweisen. Starkes sekundäres Dickenwachstum war allerdings nicht eingetreten, findet also bei den Wurzeln dieser Pflanze jedenfalls nicht in ausgiebiger Weise statt. Der exakte Nachweis, daß die Befestigungswurzeln von Aconitum japonicum ältere Stadien der Ernährungswurzeln sind, wäre allerdings erst dann erbracht, wenn an einer und derselben Wurzel der Übergang beider Typen zu verfolgen wäre, wie es bei Valeriana officinalis und anderen gelungen ist. Da die abgestorbenen Wurzeln zu kurz waren, konnte diese Frage an meinem Material nicht mit absoluter Sicherheit entschieden werden; ich zweifle aber nicht an dem entsprechenden Ergebnis der Untersuchung, die im Laufe des Sommers anzustellen wäre. Die Hauptwurzel, die die Fortsetzung der Knolle darstellt, zeigt keine Sklereiden; auffallend ist ihr großer Zentralzylinder mit vielem Parenchym und wenig Gefäßen und die kleine primäre Rinde. In einem Falle war der Durchmesser des Zentralzylinders der Hauptwurzel 130 Teilstriche des Okularmikrometers breit, während die Dicke der primären Rinde nur 11-13 Teilstriche betrug, also ungefähr den zehnten Teil des Zentralzylinders. Bei den „Ernährungsund Befestigungswurzeln", die verschieden alte Stadien der Nebenwurzeln 1. Ordnung darstellen, - starke Verzweigung findet in dem Wurzelsystem dieser Pflanze überhaupt nicht statt - betrug der Durchmesser des Zentralzylinders 15-18 Teilstriche, die Dicke der Rinde schwankt zwischen 12 und 20 Teilstrichen.

Der für die als Speicherorgane dienenden knöllchenförmig verdickten Wurzeln charakteristische Reichtum an parenchymatischen Zellen zeigt sich auch bei Ranunculus Ficaria. Die als Speicherzellen funktionierenden parenchymatischen Zellen, die dicht mit Stärke gefüllt sind, 
finden sich hier aber nicht wie bei Aconitum im Zentralzylinder, sondern in der Rinde. Der Hauptunterschied zwischen den Wurzelknöllchen und den gewöhnlichen Wurzeln bei Ranunculus Ficaria besteht in der mächtigen Entwicklung der Rinde. Doch konnte ich die von Irmisch ${ }^{1}$ ) gemachte Behauptung, daß der anatomische Bau beider Organe „im wesentlichen derselbe sei“ und die Gefäßteile bei beiden „meist deutlich getrennt sind“, nicht bestätigen. Ich fand bei den Knöllchen meist pentarchen Bau, wobei die Gefäßteile in der Mitte fast oder ganz zusammenstoßen. Die gewöhnlichen Wurzeln haben dagegen meist einen triarchen Bau und die Gefäße stoßen immer im Mittelpunkt zusammen. Bei Ranunculus Ficaria wollte ich nun auch versuchen, ob ich die knöllchenartige Form der Wurzel in die gewöhnliche umwandeln könnte. Ich pflanzte Stücke der Pflanze mit Knöllchen in Erde ein und wollte die letzteren zum Weiterwachsen und dadurch zur Umwandlung in gewöhnliche Wurzeln bringen. Leider mißlangen die Versuche, da die Pflanzen sehr bald zugrunde gingen.

Ich will die übrigen von Tschirch angegebenen Pflanzen nicht weiter besprechen, da sich überall im wesentlichen die gleichen Verhältnisse ergeben haben. Auch sind derartige Untersuchungen in der oben zitierten Arbeit von von Alten ausführlicher dargestellt. Zwei Pflanzen möchte ich aber noch besprechen, die sich zwar nicht in der Aufzählung von Tschirch befinden, die aber besonders interessante Einzelheiten zeigen und die ich näher untersucht habe. Ich meine Thalictrum aquilegifolium und Primula. Bei Thalictrum aquilegifolium lagen die Verhältnisse folgendermaßen: Die Wurzeln sind meist tetrarch. Doch kommen auch triarche und pentarche, auch hexarche vor. Es kommt jedoch auch vor, daß die Zahl der Gefäßstrahlen von der Basis nach der Spitze abnimmt; eine Wurzel z. B. (Fig. 1 u. 2) ${ }^{2}$ ) war an der Basis pentarch, nach $5 \mathrm{~cm}$ bereits tetrarch. Ich mache auf diese Tatsache, die sich auch sonst bei Pflanzen in der Natur findet, besonders aufmerksam, da es mir gelungen ist, dieselbe bei Vicia Faba experimentell hervorzurufen, worauf ich weiter unten noch eingehen werde. Im ausgewachsenen Zustande enthalten diese Wurzeln eine überraschend große Menge mechanischen Gewebes. Betrachtet man

1) Irmisch, Thilo, Beiträge zur vergleichenden Morphologie der Pflanzen, Halle 1854 (I. Ranunculus Ficaria), pag. 5.

2) Diese und die übrigen mikrophotographischen Abbildungen sind nach mit Phloroglucin-Salzsäure gefärbten Handschnitten angefertigt. Bei der Herstellung war mir Herr Dr. Wolpert behilflich, wofür ich ihm auch an dieser Stelle bestens danke. 
Untersuchungen über die Abhängigkeit der Gefäß- u. Sklerenchymbildung usw. 193

nun eine Reihe von Wurzeln, indem man Querschnitte an der Basis macht, so findet man ähnliche Unterschiede, wie sie Tschirch bei seinen Pflanzen als „Ernährungs- und Befestigungswurzeln“ abbildet.

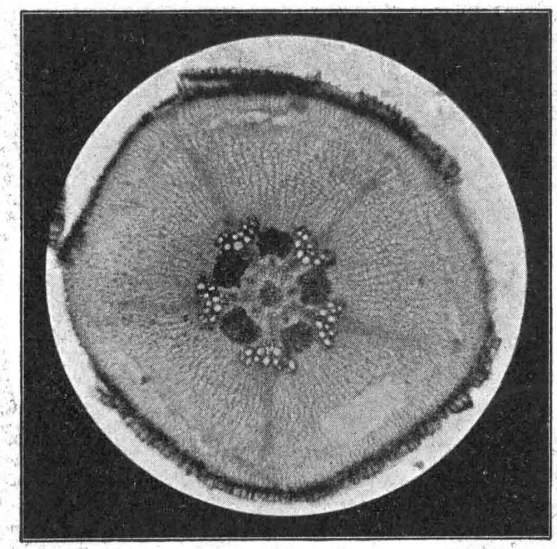

Fig. 1. Thalictrum aquilegifolium.

Wurzel an der Basis geschnitten (pentarch).

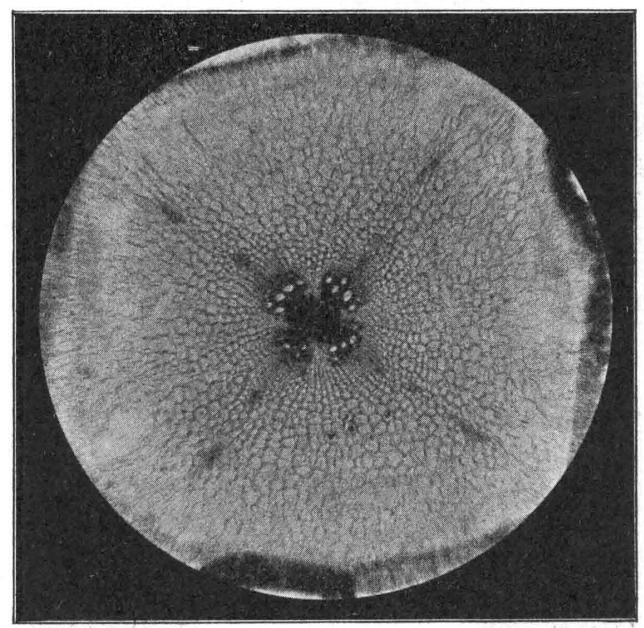

Fig. 2. Thalictrum aquilegifolium. Dieselbe Wurzel wie Fig. 1, $\tilde{\mathrm{cm}}$ tiefer (tetrarch).

Diejenigen Entwicklungsstadien, die den letzteren entsprechen, haben vor den primären Gefäßteilen, die sehr schwach entwickelt sind, mächtige Bündel mechanischen Gewebes. Bei genauerer Betrachtung zeigt sich, $\mathrm{da} B$ das letztere beim sekundären Dickenwachstum entstandenes Gewebe 
ist, das zum Holz gehört, also als Libriform bezeichnet werden muß. Das sekundäre Dickenwachstum geht nämlich bei dieser Pflanze in etwas merkwürdiger Weise vor sich. Während sich nämlich vor dem.

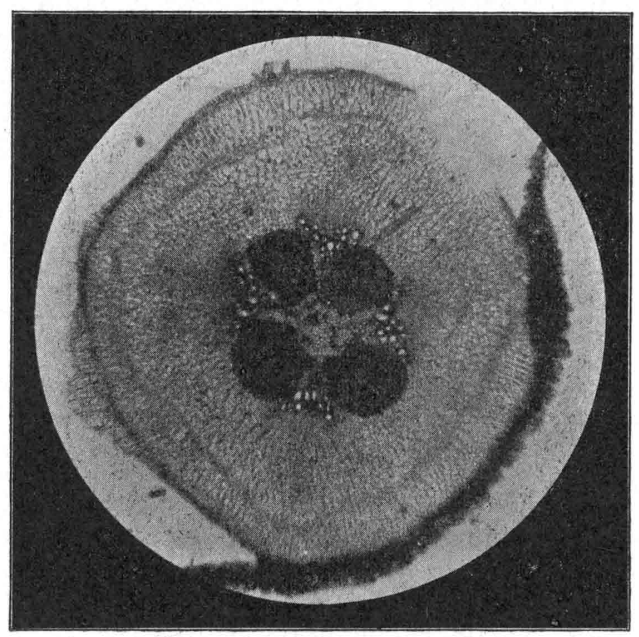

Fig. 3. Thalictrum aquilegifolium. Querschnitt einer Wurzel an der Basis.

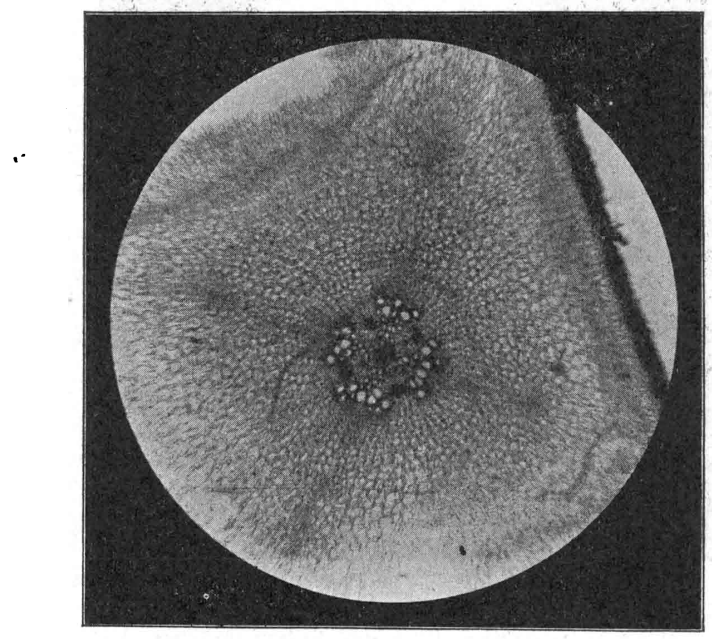

Fig. 4. Thalictrum aquilegifolium. Dieselbe Wurzel wie Fig. $3,2 \mathrm{~cm}$ tiefer geschnitten.

Vasalprimanen die eben erwähnten Libriformbündel entwickeln, entstehen zwischen denselben, also auf demselben Radius wie die Siebteile, die 
Untersuchungen über die Abhängigkeit der Gefäß- u. Sklerenchymbildung usw. 19う

Gefäße; aus den Abbildungen (Fig. 1-6) geht das deutlich hervor. Verfolgt man nun eine derartige Wurzel nach der Spitze hin, so wird die Menge des Libriforms vor den Gefäßteilen immer geringer; dafür

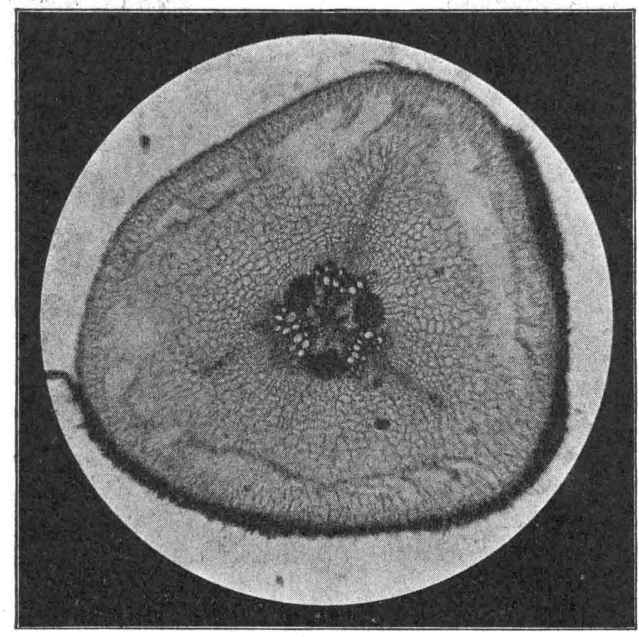

Fig. 5. Thalictrum aquilegifolium. Wurzel an der Basis geschnitten.

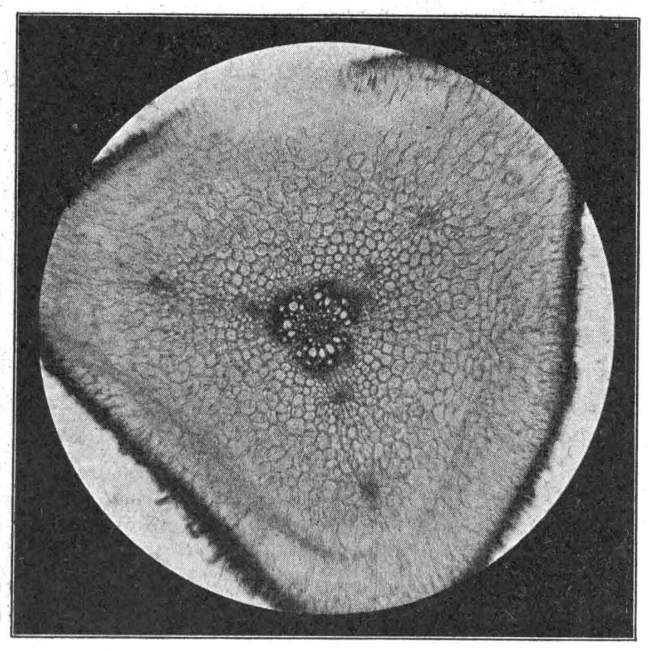

Fig. 6. Thalictrum aquilegifolium. Dieselbe Wurzel wie Fig. $5,5 \mathrm{~cm}$ tiefer.

tritt in der Mitte des Zentralzylinders Sklerenchym auf, das weiter nach unten auch verschwindet (Fig. 1 u. 2,3 u. 4, 5 u. 6). Vergleicht man 
nun verschiedene Wurzeln an der Basis, so bekommt man dieselben Bilder, die man an älteren Wurzeln in verschiedener Entfernung von

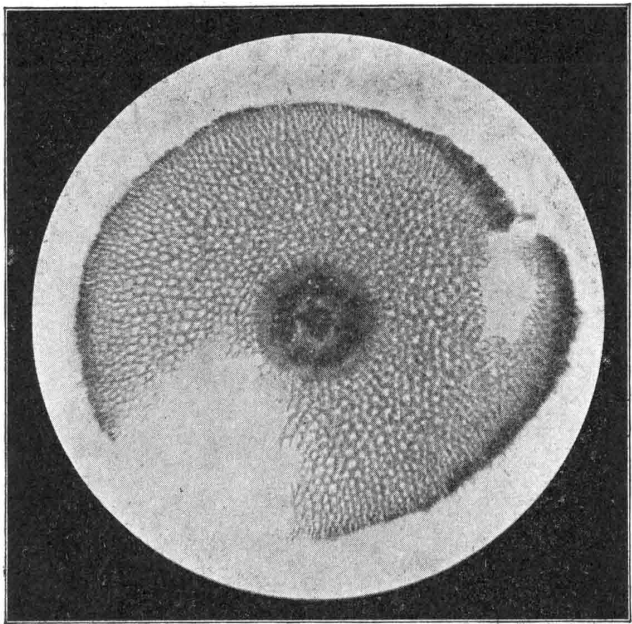

Fig. 7. Primula officinalis. Wurzel an der Basis geschnitten. „Befestigungswurzel“ im Sinne Tschirch's.

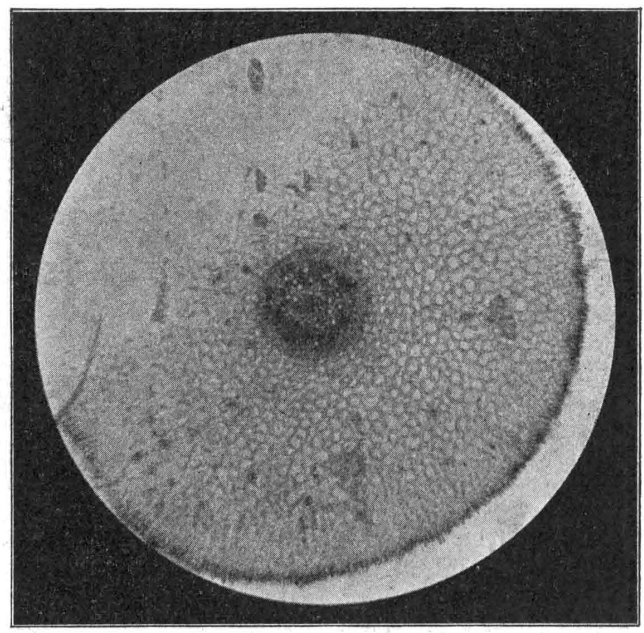

Fig. 8. Primula officinalis. Dieselbe Wurzel wie Fig. $7,1 \mathrm{~cm}$ tiefer.

- der Basis erhält. Also auch hier wieder täuschen verschieden alte Wurzeln eine Differenzierung in verschiedene Typen vor. Mit Thalic- 
trum wollte ich nun auch Versuche anstellen, um den Einfluß der Ernährungsverhältnisse zu untersuchen. Ich zog Pflanzen aus jungen Rhizomknospen und entfernte die sich entfaltenden Blätter, um die Pflanzen am Assimilieren zu verhindern, in der Erwartung, dadurch eine Reduktion der Zahl und Wandstärke der meehanischen Elemente, also der Libriformfasern, zu erhalten. Da die Pflanzen sich jedoch nur langsam bewurzelten und den operativen Eingriff der Entblätterung nicht vertrugen, gingen sie bald zugrunde. Bei Primula machte ich jedoch entsprechende Versuche, die zu einem positiven Resultate führten. Ich komme weiter unten darauf zu sprechen.

Bei der Gattung Primula liegen die Verhältnisse bei den verschiedenen Arten verschieden. Primula elatior zeigt in ausgewachsenen Wurzeln immer Sklerenchym in der Mitte des Zentralzylinders, das aber nach der Spitze zu bald aufhört. Bei meinen Wurzeln war dasselbe in einer Entfernung 2-21/2 cm von der Basis verschwunden. Noch nicht so alte Wurzeln zeigen dagegen an der Basis kein Sklerenchym oder nur eine geringe Entwicklung desselben. Ähnlich liegen die Verhältnisse bei Primula officinalis (Fig. 7-9). Auch hier hört das Sklerenchym in einer kurzen Entfernung von der Basis auf. Einige Zahlen mögen dies veranschaulichen. Eine Wurzel hatte an der Basis reichliches Sklerenchym, $1 \mathrm{~cm}$ tiefer bedeutend weniger, noch reichlich $1 \mathrm{~cm}$ tiefer hatte das Sklerenchym ganz aufgehört. Andere Wurzeln dagegen, die noch nicht so alt sind, zeigen schon an der Basis kein Sklerenchym („Ernährungswurzeln“ im Sinne Tschirch's; vgl. Fig. 10). Von den Nebenwurzeln habe ich nur wenige untersucht; sie zeigten die gewöhnlichen Reduktionserscheinungen. Ihr Zentralzylinder war diarch. Primula farinosa und Primula auricula zeigen überhaupt kein Sklerenchym. Ich verglich Exemplare von Primula auricula von feuchten Standorten (Dachauer Moor bei München) mit solchen aus dem Gebirge, in der Vermutung, daß der trockene und sonnige Standort des Gebirges die Pflanze zur Bildung von Sklerenchym veranlaßt hätte. Diese Vermutung bestätigte sich jedoch nicht. Die Pflanze ist eben $\mathrm{zu}$ wenig plastisch und die äußeren Bedingungen haben auf die Entstehung des mechanischen Gewebes weniger Einfluß als die erblichen Anlagen. Mit Primula elatior und officinalis machte ich auch einige Versuche. Ich schnitt von im Freien gesammelten Pflanzen Rhizomstücke von knapp $1 \mathrm{~cm}$ Länge, die vorn die ausgetriebene Knospe enthielten, ab und entfernte an ihnen sämtliche Wurzeln. Auch von den Blättern wurden die meisten, namentlich die größeren abgeschnitten oder wenigstens stark beschnitten, um die Pflanze vor allzu großer Transpiration zu 
schützen, da ihr die Wasseraufnahme aus dem Boden in ihrem wurzellosen Zustand fast unmöglich. gemacht wurde. Zur weiteren Herab-

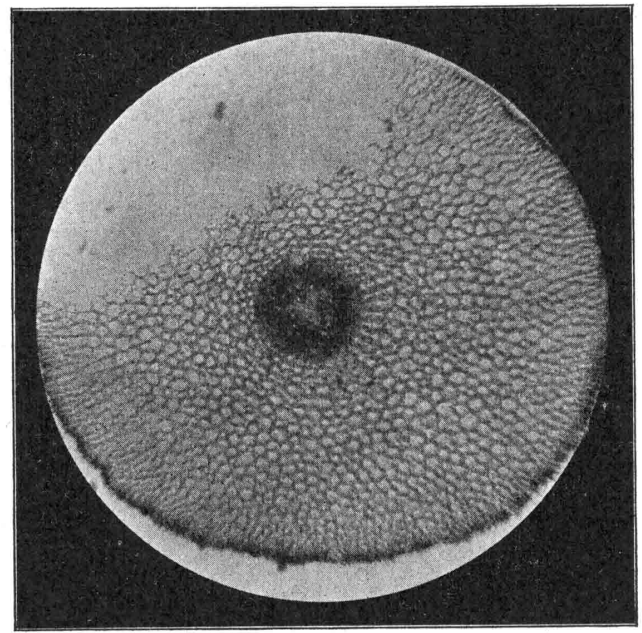

Fig. 9. Primula officinalis. Dieselbe Wurzel wie Fig. 7 und $8,2 \mathrm{~cm}$ unter der Basis.

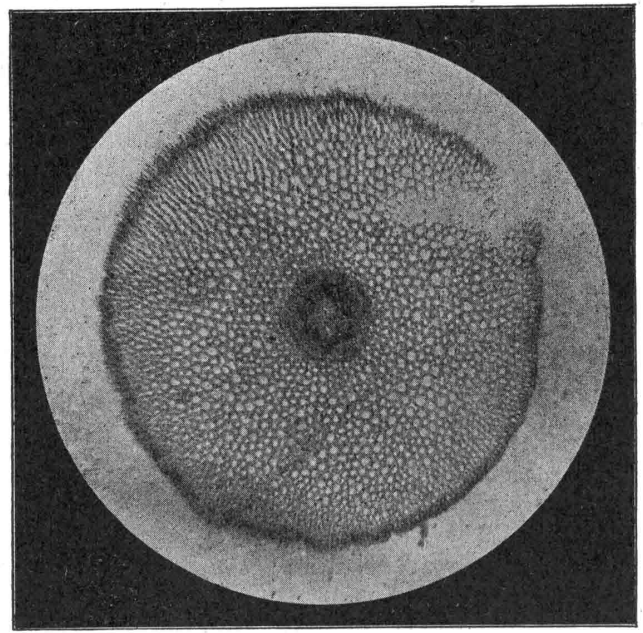

Fig. 10. Primula officinalis. Wurzel an der Basis geschnitten. „Ernährungswurzel“ im Sinne Tschirch's.

setzung der Transpiration wurden die Pflanzen wenigstens im Anfang mit einer Glasglocke bedeckt. Um ihnen im übrigen möglichst günstige 
Wachstumsbedingungen zu verschaffen, wurden die Kulturen im Frühbeet aufgestellt. Ich kultivierte sie nun fast während des ganzen Sommers unter normalen Bedingungen und außerdem bei anormal hohem Feuchtigkeitsgehalt des Bodens und bei großer Trockenheit desselben. Während sich aber herausstellte, daß die Feuchtigkeitsverhältnisse ohne Einfluß auf die Menge des Sklerenchyms blieben, war ein anderer Versuch von positivem Erfolg. Ich wollte die von dem französischen Anatomen (s. Einleitung) gefundenen Beziehungen zwischen Kohlensäureassimilation und Sklerenchymbildung prüfen, indem ich die Pflanze durch Entblättern am Assimilieren hinderte. Ich führte den Versuch in der Weise aus, daß ich alle sich entfaltenden Blätter, sobald sie eine gewisse Größe erreichten, abschnitt; die assimilierende Oberfläche war dadurch fast null geworden. In der Tat konnte ich bei diesen Kulturen, die hinter den normalen im Wachstum natürlich sehr zurückblieben, eine beträchtliche Reduktion des Sklerenchyms nachweisen. In der Mitte des Zentralzylinders, wo sich normal ziemlich beträchtliche Bündel von Sklerenchymfasern finden, war keine Spur von solchen oder nur vereinzelte zu sehen. Natürlich wurden immer Wurzeln von im übrigen gleicher Entwicklung verglichen.

Nachdem wir nun die von Tschirch behauptete Differenzierung, die er als Heterorhizie bezeichnet, kennen gelernt haben, können wir zur Kritik der oben erwähnten Wildt'schen Arbeit ${ }^{1}$ ) übergehen. Wildt legt sich die Frage vor, ob die vermeintliche Differenzierung, an der er nicht zweifelt, autonomer oder aitionomer Natur sei, d. h. ob die Anlage von Ernährungs- und Befestigungswurzeln erblich fixiert sei, etwa wie die verschiedene Ausbildung der Luftwurzeln epiphytischer Gewächse in Nähr- und Haftwurzeln ${ }^{2}$ ), oder ob beide Formen durch verschiedene äußere Faktoren hervorgerufen werden. Unter den letzteren kommt nach ihm besonders in Betracht die Einwirkung mechanischer Kräfte und die chemische Beschaffenheit des umgebenden Mediums, also des Bodens. Da nach dem vorhergehenden die Befestigungswurzeln nur ältere Stadien der Ernährungswurzeln sind, ist es selbstverständlich, daß in allen Medien, gleichgültig ob Gartenerde, Lehm, Sand oder Nährlösung, Befestigungswurzeln neben Ernährungswurzeln sich finden werden. Die Wildt'schen Versuche ergeben auch beide Formen nebeneinander in sämtlichen eben genannten Medien.

1) Wildt, Willi, Über die experimentelle Erzeugung von Festigkeitselementen in Wurzeln und deren Ausbildung in verschiedenen Nährböden. Diss., Bonn 1906.

2) Goebel, Organographie der Pflanzen, Jena 1898-1901, pag. 487. 
Da in Nährlösung eine mechanische Beanspruchung der Wurzel gänzlich ausgeschlossen ist, aber trotzdem sich der Typus der Befestigungswurzel fand, schloß Wildt, daß Befestigungswurzeln neben Ernährungswurzeln auch autonom auftreten könnten. Wildt glaubte aber auch durch mechanischen Zug Ernährungs- in Befestigungswurzeln umgewandelt zu haben. Er faßt die letzteren auf ,als Anpassungsformen an die Wirkung mechanischer Kräfte (Zugkraft)“ 1). Da wir uns aber im vorhergehenden Kapitel von der Unhaltbarkeit der Tschirch'schen Theorie der Heterorhizie überzeugt haben, muß es uns zweifelhaft vorkommen, daß der Einfluß, den der Zug nach Wildt auf die Wurzel ausübt, eine derartige Anpassung ist, ja, daß er überhaupt mit Heterorhizie, die doch auf Altersunterschiede zurückzuführen ist, etwas zu tun hat. Meine Nachprüfungen der Wildt'schen Versuche, sowie meine nachher zu erwähnenden Untersuchungen haben auch gezeigt, daß die Veränderung, die eine Wurzel durch den Zug erleidet und auf die ich gleich zu sprechen kommen werde, mit der Tschirch'schen Heterorhizie nichts zu tun hat, daß sie, un das Resultat vorweg zu nehmen, eine Hemmungsbildung darstellt. Bevor ich die Beweise meiner Behauptung anführe, will ich kurz die Methode und die Resultate der Wildt'schen Arbeit schildern. Die Wurzel wurde (Fig. 11) bei $A$ und $B$ eingegipst und zwar in der Weise, daB sie durch eine Öffnung einer Pillenschachtel durchgeführt wurde, die dann mit Gips ausgegossen wurde. Die Pillenschachtel $B$ liegt unter einem Widerlager $W$, so da $B$ sie bei longitudinalem Zuge gegen dasselbe gedrückt wird; in der Pillenschachtel $A$ ist außerdem noch eine Drahtschlinge eingegipst, an der ein Faden befestigt werden kann, der über eine Rolle $R$ geführt, am anderen Ende die Gewichte $G$ trägt. Die ganze Versuchsanordnung befindet sich bis zu den Kotyledonen in gewöhnlicher Erde. Aus der Anordnung geht ohne weiteres hervor, daß nur die Strecke zwischen $A$ und $B$ dem Zuge ausgesetzt ist, da die darüber und darunter liegenden Teile der Wurzel nicht gezogen werden. Diese Anordnung hat den Vorteil, daß gezogene und nichtgezogene Stellen an ein und derselben Wurzel verglichen werden können und individuelle Schwankungen ausgeschlossen sind. Es ist natürlich notwendig, daß sich unter $B$ nur ein kleiner Teil der Wurzel befindet, damit möglichst junges Gewebe dem Zuge ausgesetzt wird, da älteres nicht mehr in dem Maße oder gar nicht mehr umbildungsfähig ist. Das Resultat, das Wildt am Ende des Abschnittes über den Einfluß des Zuges aufstellt, daß

1) l. c. pag. 34 . 
die Wurzeln im Gegensatz zu den: Stammorganen auf Zug reagieren und sich völlig anders ausbildēn als ohne Einwirkung der Zugkraft, ist richtig, die Deutung aber, daß aus Ernährungswurzeln Befestigungswurzeln entstanden seien, ist irrig. Die Nachprüfung bei Vicia Faba besonders ergab die Richtigkeit der Wildt'schen mikrophotographischen Abbildungen. Der wesentliche Unterschied der gezogenen Strecke der nichtgezogenen gegenüber ist ein Zusammenrücken der Gefäßteile des Zentralzylinders nach der Mitte und damit verbundeh eine Reduktion des Marks. Vermehrung der mechanisch wirksamen Bestandteile, der Gefäße oder Sklerenchymfasern, oder eine Verstärkung ihręr Wandverdickungen kann Wildt nicht nachweisen. In der veränderten

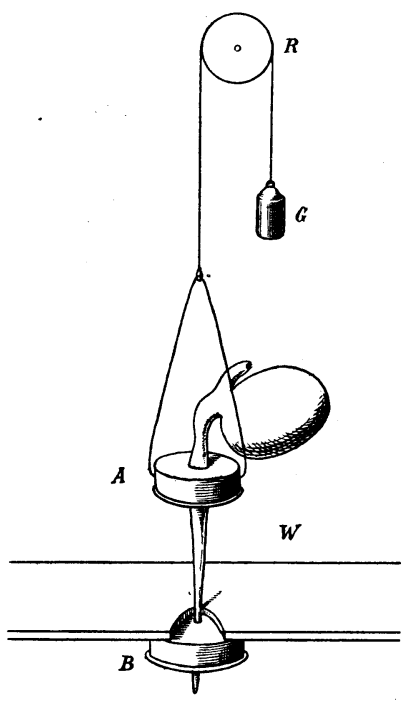

Fig. 11.
Fig. 11. Schematische Darstellung der Wildtschen Versuchsanordnung zum partiellen Zug von Wurzeln.

(Erklärung der Buchstaben im Texte.)

Fig. 12. Erklärung siehe im Texte.

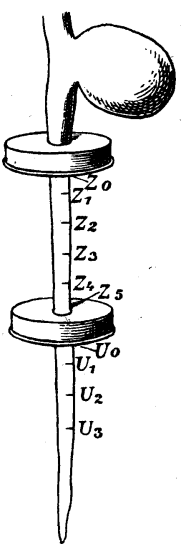

Fig. 12.

anatomischen Anordnung des Gefäßteiles erblickt er eine Anpassung an den Zug (zugfeste Konstruktion). Die Deutung der durch Zug veränderten Struktur als Anpassung, wie Wildt es will, ist ausgeschlossen. Schwendener ${ }^{1}$ ) sagt über ,zugfeste Einrichtungen“, wie schon oben erwähnt: „Die Zugfestigkeit hängt einzig und allein von der Größe des Querschnittes der widerstandsfähigen Elemente ab; die Anordnung derselben ist theoretisch vollkommen gleichgültig, sofern nur der Zug gleichmäßig auf alle widerstandsfähigen Elemente einwirkt.“ Um nun diese gleichmäßige Wirkung herzustellen, verteilt man in der Praxis

1) Schwendener, S., Das mechanische Prinzip im Bau der Monokotylen, Leipzig 1874, pag. 116. 
die mechanischen Elemente meist in der Mitte; bei der Pflanze sind „physiologische Rücksichten" maßgebend, einen mechanisch wirksamen Zentralzylinder zu schaffen, der zwecks Wasseraufnahme von der Rinde und ihrer Epidermis umgeben sein muß. Für die Zugfestigkeit ist es gleichgültig, ob die mechanisch wirksamen Bestandteile in unserem Falle hauptsächlich die Gefäße des Zentralzylinders in einem Ring angeordnet sind, der ein zentrales Mark umgibt, oder ob die Gefäßteile in der Mitte zusammenstoßen. Wenn also das Verschwinden oder wenigstens die Reduktion der Markzellen mechanisch ohne Vorteil für die Pflanze ist, so kann von einer Anpassung an die Inanspruchnahme durch Zug keine Rede sein, die ganze Erscheinung muß vielmehr als eine Hemmungsbildung betrachtet werden. Dafür will ich noch verschiedene andere Beweise liefern.

Nachgeprüft habe ich die Wildt'schen Versuche an Phaseolus und besonders an Vicia Faba; ich konnte dabei die Wildt'schen Resultate im allgemeinen bestätigen. Im folgenden will ich die aus der Kultur mit Vicia Faba gewonnenen Resultate anführen. Es handelt sich um eine Kultur, die vom 7. September bis zum 25. September, also 18 Tage gewachsen war. Ich wollte dabei auch feststellen, bis zu welcher Grenze ich die Gewichte vermehren könnte, ohne daß eine Zerreißung der Wurzel eintritt. Es gelang mir eine Steigerung derselben bis $\mathrm{zu} 2040 \mathrm{~g}$ (Wildt wandte ein Gewicht von nur $670 \mathrm{~g}$ an), bei welchem Zuge die Wurzel zerriß. Es ist selbstverständlich, daß nicht das ganze Gewicht zur Wirkung kam, daß etwa die Zerreißungsgrenze erst bei $2040 \mathrm{~g}$ liegt; die Wirkung wurde natürlich dadurch geschwächt, daß die Wurzeln mit einer großen Anzahl von Nebenwurzeln im Boden festsitzen und außerdem durch die Reibung der Erde. Über die eigentliche Zerreißungsgrenze oder die Zugfestigkeit wird weiter unten berichtet werden. Die anatomischen Querschnittsbilder der gezogenen und der nichtgezogenen Strecke ergaben Übereinstimmung mit denen von Wildt. Von einer Zunahme der mechanischen Elemente oder ihrer Wandverdickungen in der gezogenen Zone war natürlich, wie ja auch Wildt selbst zugibt, nichts zu sehen. Bezüglich der Zahl der Gefäße und Sklerenchymfasern ergaben aber genaue Zählungen, die in der folgenden Tabelle und Kurve (Fig. 13) zusammengestellt sind, Resultate, die nur die Deutung der Erscheinung als Hemmungsbildung zulassen. Zum Verständnis der in der Tabelle und Kurve gebrauchten Abkürzungen will ich noch folgende Erklärung geben. Ich bezeichne mit $O$ die Schnitte über der gezogenen Stelle, mit $U$ die unter ihnen und mit $Z$ die Schnitte innerhalb der gezogenen Stelle. Die Bedeutung der den 
Untersuchungen über die Abhängigkeit der Gefäß- u. Sklerenchymbildung usw. 203

Buchstaben beigefügten Zahlen gehen aus obiger schematischen Figur hervor (Fig. 12).

\begin{tabular}{c|c|c|c|c}
\hline \hline Schnitt & Zahl der Gefäße & $\begin{array}{c}\text { Zahl der } \\
\text { Sklerenchym- } \\
\text { fasern }\end{array}$ & $\begin{array}{c}\text { Gesamtdurchschnitt } \\
\text { der Wurzeln } \\
\text { in Millimeter }\end{array}$ & $\begin{array}{c}\text { Durchschnitt der } \\
\text { Zentralzylinder } \\
\text { in Millimeter }\end{array}$ \\
\hline $\mathbf{Z}_{0}$ & 253 & 646 & 4,32 & 2,28 \\
$\mathbf{Z}_{1}$ & 216 & 436 & 3,56 & 1,72 \\
$\mathbf{Z}_{2}$ & 188 & 348 & 3,19 & 1,51 \\
$\mathbf{Z}_{3}$ & 168 & 348 & 3,52 & 1,30 \\
$\mathbf{Z}_{4}$ & 135 & 216 & 3,25 & 1,25 \\
$\mathbf{Z}_{5}$ & 66 & 120 & 2,82 & 0,92 \\
$\mathbf{U}_{0}$ & - & 123 & 2,53 & 0,91 \\
$\mathbf{U}_{1}$ & - & 129 & 2,30 & 0,82 \\
$\mathbf{U}_{2}$ & - & 88 & 2,28 & 0,86 \\
\end{tabular}

Die erstaunlich rasche Abnahme der Sklerenchymfasern von 646 auf 120 , wie sie durch den Teil der Kurve von $A$ bis $B$ deutlich veranschaulicht wird, im Vergleich $\mathrm{zu}$ dem allmählichen Abnehmen der Sklerenchymfasern unter der gezogenen Strecke (Kurve von $B$ bis $C$ ) zeigt, wie der Zug eine hemmende Wirkung auf die Gewebsneubildung ausgeübt hat. Eine ähnliche Kurve würde sich auch zeigen, wenn man die Zahl der Gefäße berücksichtigen würde. Auch den Durchmesser sowohl der ganzen Wurzel als auch des Zentralzylinders habe ich gemessen (vgl. obenstehende Tabelle und die Kurve in Fig. 14). Es er-

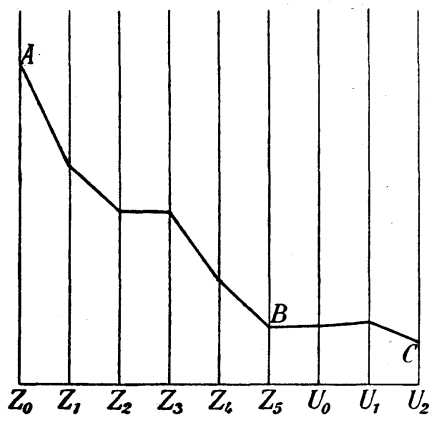

Fig. 13.

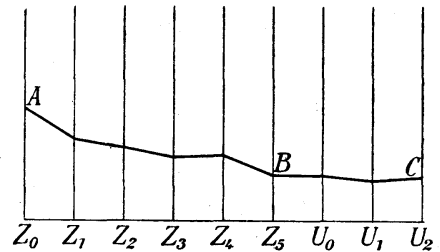

Fig. 14.

Fig. 14. Kurve zur Veranschaulichung der Abnahme der Dicke des Zentralzylinders innerhalb und unterhalb der gezogenen Strecke.

Fig. 13. Kurve zur Veranschaulichung der Abnahme der Sklerenchymfasern innerhalb und unterhalb der gezogenen Strecke.

gaben sich dabei dieselben Resultate. Am schlagendsten aber kann man wohl die Erscheinung als Hemmungsbildung nachweisen, wenn man die Zugfestigkeit der Wurzel selbst an den verschiedenen Stellen prüft. Es geschah dies in der bekannten, von Schwendener ${ }^{1}$ ) angegebenen Weise. Die Wurzel wurde oben zwischen zwei Holzklötze

1) Schwendener, l. c. pag. 9 . 
fest eingeklemmt, ebenso unten. An die unteren Holzklötze wurde eine Wagschale befestigt, auf die die Gewichte gelegt wurden. Bei diesem Versuche zeigte sich zunächst, daß die Rinde leicht vom Zentralzylinder abrutschte; ich prüfte deshalb den Zentralzylinder allein, der ja für die mechanische Leistungsfähigkeit auch nur in Betracht kommt. Die Gewichte wurden allmählich gesteigert. Es ergab sich nun, daß die gezogene Strecke einen Zug von 125 g noch aushielt, aber bei $145 \mathrm{~g}$ zerriß, die darunter liegende Strecke hielt einen Zug von $155 \mathrm{~g}$ noch aus und zerriß bei $165 \mathrm{~g}$; bei der darüber liegenden Strecke waren die entsprechenden Zahlen $235 \mathrm{~g}$ und $245 \mathrm{~g}$. $\mathrm{Daß}$ die darüber liegende Strecke zugfester ist, ist selbstverständlich, da es sich ja um älteres Gewebe handelt; daß aber die darunter liegende Strecke nicht nur keine schwächere Ausbildung zeigt, sondern sogar eine etwas stärkere, beweist deutlich, daß wir es in der gezogenen Strecke mit einer Hemmungsbildung zu tun haben.

Da das Fehlen oder die Rückbildung des Marks in den Wildtschen Versuchen eine große Rolle spielt, ebenso bei den Tschirchschen Wurzeln mit Heterorhizie, so war es mir interessant, daß ich diese Erscheinung auch auf andere Weise experimentell hervorrufen konnte. Es gelang mir das dadurch, daß ich Keimpflanzen von Vicia Faba und Phaseolus unter schlechte Ernährungsverhältnisse brachte, indem ich ihnen die großen mit Reservestoffen gefüllten Kotyledonen abschnitt. Goebel berichtet in seiner Experimentellen Morphologie ${ }^{1}$ ) von derartigen Versuchen, die nach ihm schon von Malpighi im 17. Jahrhundert angestellt wurden. Wenn er die Kotyledonen vor der Keimung abschnitt, so gingen die meisten Pflänzchen zugrunde. Ich machte mir deshalb die dort angegebene Erfahrung zunutze, daß die Entfernung der Kotyledonen in einem Alter, in dem die Wurzel der Pflanze bereits einige Zentimeter lang war, das Weiterwachsen nicht mehr hindere. Ich schildere nun die Versuchsanordnung bei Phaseolus: Keimpflanzen, deren Wurzeln eine Länge von ungefähr $6-7 \mathrm{~cm}$ erreicht hatten (die jeweilige Länge wurde immer gemessen und notiert), wurden in der angegebenen Weise behandelt und weiter kultiviert. Die Pflanzen ertrugen mit einigen Ausnahmen diese Operation, wenn ihr Aussehen auch ein kümmerliches war im Vergleich zur normalen Kontrollkultur. Während von den Keimpflanzen ohne Kotyledonen kaum die gegenständigen, ungeteilten Primärblätter entfaltet waren, hatte die Kontrollpflanze schon einige dreigeteilte, kräftige Folgeblätter getrieben; nicht

1) Goebel, Einleitung in die experimentelle Morphologie der Pflanzen. Leipzig u. Berlin 1908, pag. 16. 
also die in den Kotyledonen aufgespeicherten Reservestoffe allein, sondern auch die baldige Möglichkeit zu kräftiger Assimilation hatten die Kontrollpflanzen vor den operierten voraus. Der Hemmung in der äußeren Entwicklung entsprach auch eine Hemmung der anatomischen Ausbildung der Wurzeln, die allein untersucht wurden. Die Hemmung war eine doppelte, eine auffallende Verringerung der Zahl der Gefäße und was besonders interessant ist, eine Reduktion der Markzellen bis zu deren völligem Verschwinden. Ich will die Resultate einer Kultur beschreiben. Es handelt sich um eine Kultur, die am 27. April angesetzt und am 7. Juni ausgetopft wurde. Eine Pflanze hatte während dieser Zeit außer den beiden ungeteilten gegenständigen Primärblättern nur zwei dreiteilige Folgeblätter ganz und eines fast entfaltet. Die primären Blätter waren bei fast allen. Exemplaren etwas verdorrt, ein Beweis für die geringe Widerstandsfähigkeit dieser Pflanzen. Das Wurzelsystem zeigte bei diesen Pflanzen eine auffallend geringere Verzweigung als bei den Kontrollpflanzen. Um die Reduktion in der Zahl der Gefäße zu beweisen, bringe ich folgende Tabelle:

$\begin{array}{cccc}\begin{array}{c}\text { Zentimeter } \\ \text { von der Spitze }\end{array} & \begin{array}{c}\text { Ohne } \\ \text { Kotyledonen }\end{array} & \text { Normal } & \begin{array}{c}\text { Zentimeter } \\ \text { von der Spitze }\end{array} \\ 1 & 16 & 46 & 1 \\ 6 & 17 & 38 & 5 \\ 11 & 29 & 64 & 10\end{array}$

Wenn die Reduktion auch nicht überall so weit vorschreitet wie hier, so ist eine Reduktion gegenüber den normalen Pflanzen unverkennbar. Wichtiger als diese ist jedoch für unsere Betrachtungen die Reduktion der Markzellen. Ich gebe wieder eine Beschreibung der Ergebnisse. Die Pflanzen, deren Kotyledonen in einem Stadium abgeschnitten wurden, in dem die Wurzeln eine Länge von $5-7 \mathrm{~cm}$ besaßen, wurden nicht sehr lange weiter kultiviert und ergaben folgendes Resultat. Der Zuwachs der Wurzel betrug nur wenige Zentimeter, gewöhnlich 1-2 cm. Es konnte an diesem Material an der Spitze in der zugewachsenen Zone deutlich eine Reduktion der Markzellen bis zu deren völligem Verschwinden konstatiert werden. Man kann allgemein sagen, daß nach ungefähr $1 \mathrm{~cm}$ das Mark verschwunden ist. Es drängte sich mir die Frage auf, ob diese Hemmung der Markzellenbildung aufhören würde, sobald die Pflanze wieder unter günstige Ernährungsverhältnisse gebracht würde. Das letztere mußte ja im Laufe der Entwicklung von selbst entstehen, sobald sich eine genügende Menge Blätter entfaltet hatten, die als Assimilationsapparat, die Pflanze unabhängig von den Reservestoffen des Samens machten. Zu diesem Zwecke wurden die Versuche nochmals in größerem Maßstabe und 
längere Zeit hindurch ausgeführt. Ich gebe zunächst eine genaue Beschreibung der Versuche. Eine Keimpflanze, deren Wurzel die Länge von nicht ganz $6 \mathrm{~cm}$ erreicht hatte, wurde ihrer Kotyledonen beraubt. Am Ende des Versuchs, der fast 6 Wochen dauerte, betrug die Länge der Wurzel $26 \mathrm{~cm}$. Ich untersuchte die Wurzel nun, indem ich von der Basis nach der Spitze zu von Zentimeter zu Zentimeter Schnitte machte. Bis zu $5 \mathrm{~cm}$ zeigte sich dabei das gewöhnliche Bild einer Phaseoluswurzel: ein tetrarcher Zentralzylinder mit reichlichem Mark in der Mitte. Schon bei $6 \mathrm{~cm}$ oder etwas darüber zeigte sich eine Reduktion des Marks bis auf Spuren, bei $7 \mathrm{~cm}$ war dasselbe vollständig verschwunden. Die darunter liegenden Schnitte ergaben immer dasselbe Bild der gehemmten Gewebeausbildung bis zu einer Entfernung von $20 \mathrm{~cm}$ von der Basis, wo dann wieder deutlich Mark auftritt, das auch bis zur Spitze erhalten bleibt.

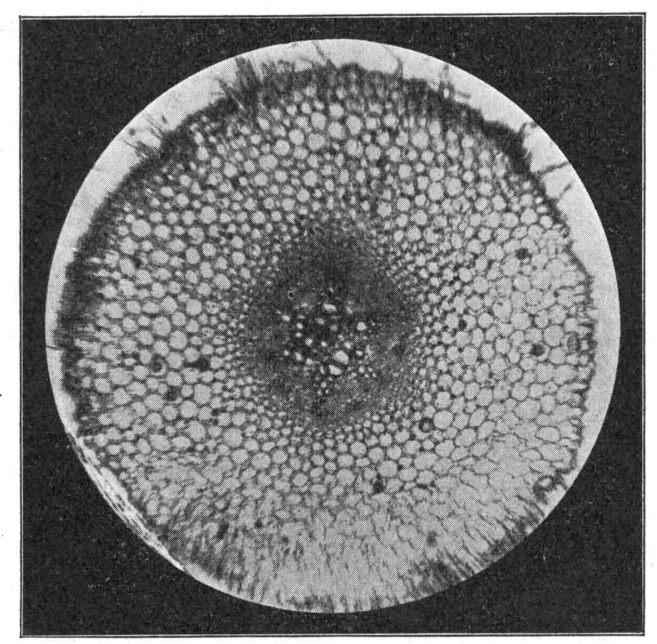

Fig. 15. Vicia Faba, Wurzelquerschnitt. Die Markzellen im Zentralzylinder sind verschwunden infolge des Abschneidens der Kotyledonen.

Diese Hemmungserscheinung stimmt vollständig überein mit dem Ergebnis der Wildt'schen Arbeit. Daß die durch Zug bewirkte Veränderung der Gewebe, die Wildt als Anpassung an den Zug auffaßt, eine typische Hemmungsbildung ist, geht daraus besonders deutlich hervor. Etwas ähnliches ergaben die Experimente mit Vicia Faba. Nach ungefähr $1 \mathrm{~cm}$ Zuwachs fand auch hier ein Verschwinden der Markzellen statt. Ich will eine Abbildung (Mikrophotographie) einer 
Wurzel mit reduziertem Mark geben wegen der Übereinstimmung mit den bei dem Wildt'schen Zugversuche erhaltenen Bildern (Fig. 15). Noch etwas Bemerkenswertes konnte ich bei diesem Experimente an Vicia Faba konstatieren, nämlich eine Reduktion der Gefäßstrahlen im Zentralzylinder. Während die Reduktion der Markzellen abèr schon nach ungefähr $1 \mathrm{~cm}$ eintritt, findet die letztere erst nach ungefähr $3 \mathrm{~cm}$ statt, wieder ein deutlicher Beweis, wie die Zahl nicht nur der Gefäße, sondern auch der Gefäßstrahlen von Ernährungsverhältnissen abhängt. Die Reduktion der Gefäßstrahlen trat jedoch nicht immer auf. Besonders aber dann, wenn die. Wurzel hexarch war; sie wurde dann pentarch und blieb nach der Spitze zu so oder wurde schließlich tetrarch. Bei Phaseolus hatte ich etwas derartiges nicht konstatieren können; es ist dies jedenfalls darin begründet, daß deren Wurzeln ziemlich konstant tetrarch sind; äußerst selten trifft man eine pentarche. Ein Auseinanderweichen der Gefäßstrahlen und Bildung von Mark dazwischen, also ein Rückgängigmachen der Hemmungserscheinung, trat bei Vicia Faba weiter nach der Spitze zu ziemlich bald ein, eher als bei Phaseolus, bei einer Wurzel z. B. nach $5-6 \mathrm{~cm}$. Im Anschluß an die in meinem Experiment aufgetretene Reduktion des Marks im Zentralzylinder möchte ich darauf hinweisen, daß diese Erscheinung auch in einer anderen meiner Kulturen, scheinbar unabhängig von äußeren Faktoren aufgetreten ist. Ich kultivierte zu einem bestimmten Zwecke Vicia Faba in Wasserkultur; bei einigen Pflanzen derselben war das Mark der Wurzel auf einige Zentimeter unterdrückt. Nach obigem Resultate handelte es sich auch hier wohl zweifellos um Störung in den Ernährungsverhältnissen, deren Art sich im einzelnen nicht weiter erkennen ließ.

Bei Vicia Faba achtete ich noch auf das Verhalten eines anderen Gewebes bei meinen operierten Pflanzen, nämlich des Sklerenchyms. Eine normale Vicia Faba fängt nämlich einige Zentimeter, meist 3-5, von der Spitze der Wurzel entfernt, an, Sklerenchym zu bilden, das nach oben rasch an Zahl seiner Elemente sowie Stärke der Wandverdickungen zunimmt. Bei den operierten Pflanzen dagegen begann die Sklerenchymbildung erst viel später. Ein Beispiel möge das zeigen. Die Wurzel, die ich jetzt im Auge habe, hatte noch bei $17 \mathrm{~cm}$ keine SSpur von Sklerenchym, bei $18 \mathrm{~cm}$ waren in einigen Bastsicheln einzelne Sklerenchymfasern ausgebildet, nach der Basis nahm das Sklerenchym zu, aber viel langsamer als bei normalen Pflanzen. Für die Abhängigkeit der Sklerenchymbildung von Ernährungsverhältnissen ist also hier ein deutliches Beispiel gegeben. Das gleiche Resultat der Rückbildung 
des Sklerenchyms konnte ich auf andere Weise erreichen, ebenfalls durch ungünstige Ernährungsverhältnisse. Ich verhinderte Pflanzen von Vicia Faba an der Kohlensäureassimilation, und zwar auf zweifache Weise, einmal, indem ich sie im Dunkeln zog, das andere Mal, indem ich ihre assimilierende Oberfläche auf ein Minimum beschränkte durch Abschneiden der Blätter (der Chlorophyllgehalt der Stammoberfläche kommt im Vergleich zu dem der Blätter kaum in Betracht). Das Resultat war in beiden Fällen das gleiche, woraus hervorgeht, daß bei den etiolierten Pflanzen der Mangel an Licht nicht direkt die Sklerenchymbildung hemmt, sondern indirekt durch Beeinflussung der Assimilation und damit der Ernährung überhaupt. Die etiolierten Kulturen wurden in der Weise angesetzt, daß in Sägemehl angekeimte Samen, deren Blätter noch nicht entfaltet waren, in Gartenerde gebracht wurden und nun unter ständiger Verdunkelung kultiviert wurden, bis ihre Wurzeln eine Länge von $16-25 \mathrm{~cm}$ erreicht hatten. Einige Zahlen mögen die Resultate erläutern. Bei einer Wurzel, die eine Länge von $16 \mathrm{~cm}$ erreicht hatte, begann die Sklerenchymbildung schon bei $5 \mathrm{~cm}$, bei einer Wurzel mit einer Länge von $21 \frac{1}{2} \mathrm{~cm}$ aber erst zwischen $10-11 \mathrm{~cm}$, bei einer Wurzel mit einer Länge von $19 \mathrm{~cm}$ in ähnlicher Entfernung. Es sind diese Reduktionen ja bei weitem nicht so groß wie jene, die durch Entfernung der Kotyledonen erreicht wurden; das Beschneiden der Kotyledonen ist eben eine in das Leben der Pflanze tiefer eingreifende Schädigung. Trotzdem wurden auch bei den etiolierten Pflanzen solche gefunden, bei denen das Mark eine Reduktion erfahren hatte, wenn auch nicht so auffallend wie in den oben besprochenen Kulturen. Auch eine Reduktion der Gefäßstrahlen und der Gefäße überhaupt konnte beobachtet werden, wenn auch nicht mit der Regelmäßigkeit wie in den genannten Kulturen. In der Absicht, die von Wildt gefundenen Gewebeveränderungen der Wurzeln durch Zug, die ich als Hemmungsbildung erkannt und bewiesen habe, auf eine noch weitere Basis $\mathrm{zu}$ stützen, unternahm ich noch einige Versuche, die ebenfalls eine Hemmung der Gewebe erwarten ließen, z. B. gipste ich die wachsende Zone der Wurzeln ein. Es geschah dies in der Weise, da $B$ ich die Wurzeln der Pflanzen - es handelte sich wieder um Vicia Faba und Phaseolus - mit Ausnahme eines knapp $1 \mathrm{~mm}$ großen Stückes der Wurzelspitze in einen Gipsmantel einhüllte, der sich bis auf einige Zentimeter hinter der Spitze erstreckte. Das Wichtigste, das sich bei diesem Versuche ergab, war zunächst eine Reduktion der Zahl der Gefäße, nicht aber der Gefäßstrahlen und des Zentralzylinders. Einige Zahlen mögen dies erläutern: 
Untersuchungen über die Abhängigkeit der Gefäß- u. Sklerenchymbildung usw. 209

\begin{tabular}{|c|c|c|c|c|}
\hline & & & $\begin{array}{l}\text { Zahl } \\
\text { der Gefäße }\end{array}$ & $\begin{array}{l}\text { Dicke der Zentralzylinder } \\
\text { in Teilstr. d. Okularmikrosk. }\end{array}$ \\
\hline Wurzel I. & $\left.\begin{array}{l}\text { oberhalb } \\
\text { innerhalb } \\
\text { unterhalb }\end{array}\right\}$ & d. Eing. & $\begin{array}{l}340 \\
137 \\
146\end{array}$ & $\begin{array}{c}126 \\
73 \\
83^{1 / 2}\end{array}$ \\
\hline Wurzel II. & $\begin{array}{l}\text { oberhalb } \\
\text { innerhalb } \\
\text { unterhalb }\end{array}$ & d. Eing. & $\begin{array}{r}233 \\
132 \\
133\end{array}$ & $\begin{array}{l}94 \\
61^{1} / 2 \\
84^{1} / 2\end{array}$ \\
\hline
\end{tabular}

(Die Zahlen sind einer Kultur von Vicia Faba entnommen).

Es ergibt sich aus dieser Tabelle, daß die Zahl der Gefäße, die in der eingegipsten Zone eine Hemmung erfahren haben, unterhalb dieser Zone nur wenig oder fast gar nicht zunimmt. Anders der Zentralzylinder: er dehnt sich nach der in der eingegipsten Zone erlittenen Einschnürung gleich wieder aus, wenn auch nicht sofort auf das ursprüngliche Maß. Dieses Resultat der Verringerung der Zahl der Gefäße durch Eingipsung, also durch mechanische Hemmung steht in Widerspruch mit den Ergebnissen einer Arbeit von Rudolf Prein 1),

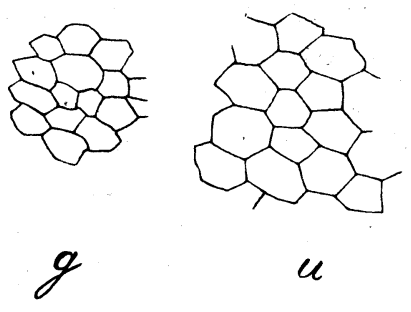

Fig. 16.

Fig. 16. Zellen aus dem Zentralzylinder der Wurzel von Phaseolus; $g$ aus der eingegipsten Zone, $u$ aus der darunter befindlichen Zone.

Fig. 17. Zellen aus dem Zentralzylinder der Wurzel von Vicia Faba; $g$ aus der eingegipsten, $o$ aus der darüber, $u$ aus der darunter befindlichen Strecke.
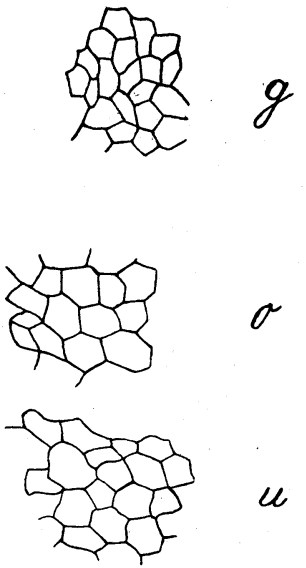

$u$

Fig. 17.

die sich mit derartigen Versuchen beschäftigt. Allerdings experimentierte der Verfasser mit einer anderen Pflanze, nämlich einer Varietät von Raphanus sativus. Er fand bei seinen Versuchen Konstanz der Zahl der Gefäße trotz mechanischer Hemmung, die er dadurch erzeugte, daß er seine Pflanzen zwischen zwei Schieferplatten oder in einer engen Glasröhre wachsen ließ. Eine andere Erscheinung, die Prein bei seinen Hemmungsversuchen beobachtete, konnte ich bei meinen Kulturen auch beobachten, nämlich ein Kleinerbleiben der Zellen in der mechanisch

1) Prein, Rudolf, Über den Einfluß mechanischer Hemmungen auf die histologische Entwicklung der Wurzeln. Inaug.-Diss., Bonn 1908. 
gehemmten, bei mir also eingegipsten Zone. Die Figuren (Fig. 16 u. 17) mögen dies veranschaulichen.

Da sich die Entfernung der im Samen aufgespeicherten Reservestoffe als ein tief eingreifender Faktor erwiesen hatte, der eine beträchtliche Hemmung der Gewebeausbildung bewirken konnte, versuchte ich diese Methode auch einmal auf eine monokotyle Pflanze auszudehnen, und zwar auf Zea Mays. Es konnte sich hierbei natürlich nicht um Entfernung der Kotyledonen handeln, sondern um eine Entfernung des dieselben physiologisch vertretenden Endosperms. Bei einer im Sommer angesetzten Kultur ergab sich nun folgendes: Schon der äußere Habitus zeigte wieder wie bei den Kulturen von Vicia Faba und Phaseolus die Hemmung in der Entwicklung. Das Endosperm wurde entfernt, nachdem die Hauptwurzel eine Länge von $3^{1 /} / 2-4^{1} / 2 \mathrm{~cm}$ erreicht hatte und außerdem sich ungefähr $2-3$ Nebenwurzeln von $2-4 \mathrm{~cm}$ Länge entwickelt hatten. Nach Beendigung des Versuchs war die Hauptwurzel der operierten Pflanze nur wenig gewachsen, bis zur Länge von ungefähr $10 \mathrm{~cm}$, während die Kontrollkulturen solche von $65 \mathrm{~cm}$ aufwiesen. Dasselbe zeigte. sich in der Länge und Zahl der Neben- und Adventivwurzeln. Die Unterschiede in den beiden Kulturen erstreckten sich sowohl auf Hauptwurzel als auch auf Neben- und Adventivwurzeln. In der Wurzel sah man vor allem eine geringere Verdickung der Endodermis, die sich mit Phloroglucinsalzsäure rot färbte und der die Gefäße umgebenden Zellen. Die Zahl der Gefäße und Gefäßstrahlen schwankt bei Zea Mays zu sehr, als daß man daraus sichere Schlüsse hätte ziehen können, auch schienen die Veränderungen keine so bedeutenden zu sein, wie bei den untersuchten Dikotylen, da ja bei den Gräsern das Wurzelsystem durch Adventivwurzeln bald ein sehr reiches wird und die Hemmung sich deshalb vielleicht mehr in einer Reduktion der Zahl und Länge der Wurzeln als der Histologie derselben ausdrücken wird.

Nachdem ich nun so durch vergleichende Beobachtung die Haltlosigkeit der von Tschirch behaupteten Differenzierung in Ernährungsund Befestigungswurzeln festgestellt hatte und damit die Grundlage für die Wildt'schen Experimente hinfälhg geworden waren, die letzteren aber auch durch meine Untersuchungen als Hemmungsbildung und nicht als Anpassung an mechanische Inanspruchnahme gedeutet worden waren, blieb als Beispiel einer Aktivitätshypertrophie noch der von Vöchting gefundene Fall an Fruchtstielen von Cucurbita Pepo übrig. Auch V öchting ist durch die Untersuchungen von Wiederstein und Ball zu der Überzeugung gekommen, daß die von Hegler behauptete Anpassung an den 
Zug durch qualitative und quantitative Vermehrung der mechanisch wirksamen Zellelemente irrig sei. In seiner experimentellen Anatomie ${ }^{1}$ ) nun schildert er einen Versuch, aus dem er die Abhängigkeit der Bildung mechanischer Zellen von Korrelationsverhältnissen schließt. Durch Unterdrückung der Geschlechtstätigkeit bei Phyllocactus und Wirsing erhielt er hypertrophische Achsen, die sich durch den Mangel an Sklerenchymelementen auszeichneten. Pflanzte er nun in solche hypertrophische Pflanzen ein Reis einer normalen Pflanze ein, so bekam die erstere normales Gewebe mit Sklerenchym. Da aber die Korrelationsverhältnisse letzten Endes auch wieder auf Ernährungsverhältnissen beruhen, so stimmen diese Resultate mit den obigen Ausführungen und Anschauungen überein. Vöchting geht nun von dieser Abhängigkeit der Sklerenchymbildung von Korrelationen aus und stellt sich die Frage, ob nicht auch Korrelationen anderer Art dasselbe hervorrufen könnten. Er sagt ${ }^{2}$ ): „Also eine dem Körper angehängte beliebige Last hat keinen oder nur geringen Einfluß auf den fraglichen Vorgang; gilt dasselbe von dem Eigengewichte? Wäre es nicht möglich, daß der Körper dies als Reiz empfände und infolgedessen mechanische Zellen erzeugte?" Der Gedanke ist sehr befremdend. Denn es ist nicht recht einzusehen, inwiefern ein Gewicht, das ich äußerlich an die Pflanze anhänge, anders auf dieselbe wirken sollte, als das Eigengewicht eines Organs. Mit den mit Ernährungsverhältnissen zusammenhängenden Korrelationen hat dies nichts zu tun, wie Vöchting meint. Aus der Nachprüfung der Wildt'schen Versuche hat sich ja sogar eine Schädigung und Hemmung der Gewebeausbildung in manchem Falle bei Zug ergeben. Doch gehen wir zur Erörterung und Nachprüfung des Vöchting'schen Versuches über. Er kultivierte Früchte von Cucurbita Pepo an der Erde liegend und freihängend. Bei letzterer fand er eine quantitative und qualitative Zunahme der mechanisch wirksamen Zellen. Die Versuchsanordnung ist aber, wie man nach einigem Überlegen erkennen wird, nicht einwandfrei. Denn in den beiden Parallelversuchen ist nicht nur die mechanische Inanspruchnahme eine verschiedene, sondern auch die Ernährungsverhältnisse sind es. Es ist ohne weiteres verständlich, daß der auf der Erde liegende Kürbis infolge des Feuchtigkeitsgehaltes der Erde und der ihn beschattenden Blätter in der Transpiration und Assimilation bedeutend gehemmt ist im Vergleich zu dem freihängenden, zu dem die Sonnen-

1) Vöchting, Hermann, Untersuchungen zur experimentellen Anatomie und Pathologie des Pflanzenkörpers. Tübingen 1908, pag. 283.

2) Vöchting, l. c. pag. 286 . 
strahlen ungehemmten Zutritt haben und ebenso der die Transpiration befördernde Wind. Von den beiden Faktoren, der Transpiration und Assimilation, scheint mir der erstere der weitaus wichtigere, wenn nicht maßgebende zu sein. Es wird dies aus den weiter unten angeführten Experimenten hervorgehen. Von diesen Erwägungen ausgehend, stellte ich folgende Versuche mit Kürbissen an. Ich kultivierte wie Vöchting einen Kürbis unten an der Erde, einen in der Höhe freihängend, einen dritten in der Höhe, aber unterstützt. War nun die Vöchting'sche Anschauung richtig, daß eine Pflanze, wenn sie das Gewicht eines Organs zu tragen hat, mit dem sie für gewöhnlich nicht belastet ist, mit einer Verstärkung ihres Sklerenchyms antwortet, so mußte sich ein Unterschied zeigen einerseits zwischen dem am Boden liegenden und dem in der Höhe befindlichen unterstützten, andererseits dem in der Höhe freihängenden. Waren jedoch die Ernährungsverhältnisse maßgebend, so mußten die beiden in der Höhe befindlichen, der unterstützte sowohl wie der freihängende, gleich ausgebildet sein und der an der Erde liegende davon verschieden. Leider gingen die am Boden liegenden Früchte zugrunde oder reiften nicht ganz aus wegen der Ungunst der Witterung des Sommers 1909. Doch kann ja über die Richtigkeit der Vöchting'schen Theorie auch an den beiden in der Höhe befindlichen Früchten, der unterstützten und der freihängenden, entschieden werden. Wenn Vöchting recht hatte, so mußten die beiden verschieden ausgebildet sein, waren jedoch die Ernährungsverhältnisse maßgebend, so mußten beide gleich sein. Diese beiden Früchte wuchsen zu ziemlich kräftigen Exemplaren heran. Am 6. Oktober wurden sie abgenommen. Der freihängend kultivierte wog mit Stiel 5,542 kg, davon betrug das Gewicht des Stieles $42 \mathrm{~g}$; der unterstützt kultivierte wog mit Stiel $4,597 \mathrm{~kg}$, der Stiel allein $65,5 \mathrm{~g}$. Ehe ich $\mathrm{zu}$ den Resultaten der anatomischen Untersuchung übergehe, möchte ich kurz die Vöchting'schen Angaben besprechen. Dieser Forscher behauptet, daß der an der Erde kultivierte und der freihängende Kürbis sich unterscheiden durch die verschieden starke Ausbildung der Wandverdickungen, einerseits der parenchymatischen Elemente zwischen den einzelnen Gefäßbündeln und zwischen dem rindenständigen Siebteil der Gefäßbündel und dem Kollenchymringe, der sich unter der Rinde befindet ${ }^{1}$, andererseits der Sklerenchymfasern in den Bastsicheln. Da bei meinen Exemplaren die eben genannten parenchymatischen Elemente

1) Über die anatomischen Einzelheiten im Bau des Fruchtstieles der Cucurbitaceen vergleiche A. Fischer, Untersuchungen über das Siebröhrensystem der Cucurbitaceen. Berlin 1884, pag. 77 und Taf. VI. 
Untersuchungen über die Abhängigkeit der Gefäß- u. Sklerenchymbildung usw. 213

keine nennenswerten Wandverdickungen hatten, sondern von ziemlich zartwandiger Beschaffenheit waren - es handelte sich bei mir zweifellos um eine andere Rasse - so blieben zum Vergleiche vor allem die Sklerenchymfasern übrig. Diese weisen nun bezüglich ihrer Wandverdickungen bei Vöchting ziemlich erhebliche Unterschiede auf; man vergleiche Fig. 14 und 19 auf Taf. X. Ich habe bei meinen zwei Kürbissen ebenfalls auf die Wandverdickungen der sklerenchymatischen Elemente geachtet und habe sie auch gezeichnet. Fig. 18 stellt einige Stellen aus dem Sklerenchym des freihängend, Fig. 19 aus dem Sklerenchym des unterstützt kultivierten Kürbisses dar. Eine stärkere Verdickung auf Seiten des Kürbis, der sein eigenes Gewicht tragen mußte, ist nicht im geringsten wahrzunehmen. Man könnte beim Betrachten der Zeichnungen eher zu dem Schlusse kommen, daß die Stärke der Verdickung bei dem unterstützt kultivierten Kürbis eine größere sei.

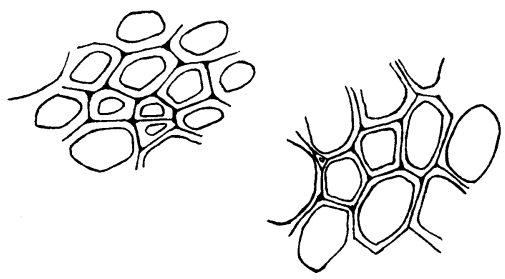

Fig. 18.
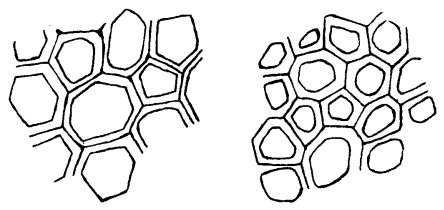

Fig. 19.

Fig. 18. Zellen aus zwei verschiedenen Bastsicheln des Stieles der freihängend kultivierten Frucht von Cucurbita Pepo.

Fig. 19. Zellen aus zwei verschiedenen Bastsicheln des Stieles der unterstützt kultivierten Frucht von Cucurbita Pepo.

Doch schwankt dieselbe an den verschiedenen Stellen einer Bastsichel und in den verschiedenen Sicheln zu sehr, als daß man daraus sichere Schlüsse ziehen könnte. Ich versuchte deshalb die Ausdehnung, also die Menge des Sklerenchyms in den beiden Fruchtstielen zu vergleichen. Es geschah dies in der folgenden Weise. Ich färbte die Schnitte mit Phloroglucinsalzsäure, um die Bastsicheln deutlich hervortreten zu lassen und zeichnete sie dann einzeln bei schwacher Vergrößerung - bei starker Vergrößerung wäre eine einzelne Sichel nicht in ihrer ganzen Ausdehnung ins Gesichtsfeld des Mikroskops gekommen. Die so erhaltenen Zeichnungen wurden dann mit Hilfe eines Pantographen vierfach vergrößert, auf sehr starkes Papier übertragen, ausgeschnitten und gewogen. Die Wägung ergab für den freihängend kultivierten Kürbis $5,9 \mathrm{~g}$, für den unterstützt kultivierten $10 \mathrm{~g}$. Die Untersuchung ergibt also ein überraschendes Resultat; nach der Vöchting'schen Anschauung sollte man das Gegenteil erwarten. Die Unhaltbarkeit der 
letzteren geht daraus mit besonderer Deutlichkeit hervor. Auffallend bleibt aber immerhin der große Unterschied in der Menge des Sklerenchyms in den beiden Fruchtstielen (in dem einen Falle fast das doppelte wie in dem anderen). Da nach dem vorhergehenden mechanische Faktoren ausgeschlossen sind, müssen wir nach anderen Ursachen suchen. Am nächsten liegt es ja, an das Vorhandensein individueller Schwankungen zu denken. Beim Betrachten der Maße der Fruchtstiele, die in folgender Tabelle zusammengestellt sind, fiel mir die bedeutend größere Länge des Fruchtstieles der freihängend kultivierten Frucht auf.

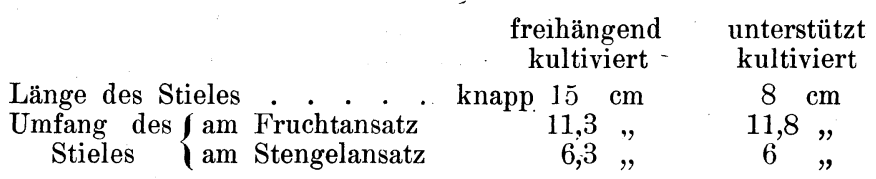

- Bildet man nun das Verhältnis der Länge des Stieles des freihängend gezogenen Kürbis zu der des unterstützt gezogenen, also $15 \mathrm{zu} 8$, so ergibt sich die Zahl $1,8-1,9$; bildet man dann das Verhältnis der Menge des Sklerenchyms in der unterstützt gezogenen Frucht zu der in der freihängend gezogenen Frucht, also $10 \mathrm{zu} \mathrm{6}$, so ergibt sich eine ähnliche Zahl, nämlich 1,7. Da nun der Umfang des Stieles bei beiden Früchten ungefähr der gleiche ist, wie sich aus obiger Tabelle erkennen läßt, so ist das Verhältnis der Längen identisch mit dem der Massen der beiden Stiele. Um so viel größer bei dem einen Stiel die Masse ist, so viel kleiner also ist die Menge des Sklerenchyms auf dem Querschnitt, oder die Gesamtmasse des Sklerenchyms ist bei beiden Stielen gleich groß. Diese Deutung hat viel Wahrscheinlichkeit für sich; so viel geht aber aus den obigen Versuchen und Messungen mit unzweifelhafter Klarheit hervor, daß die Vöchting'sche Anschauung, daß eine Pflanze, die das Gewicht eines Organs zu tragen hat, mit dem sie für gewöhnlich nicht belastet ist, mit einer Verstärkung ihres mechanischen Apparates antwortet, unhaltbar ist und daß die geringere Ausbildung des Sklerenchyms des am Boden liegenden Kürbis in dem Vöchting'schen Versuche als Hemmungsbildung zu erklären ist, bedingt durch die geschwächte Transpiration.

Um den Einfluß der Ernährungsverhältnisse noch mehr zu prüfen, stellte ich noch andere Versuche an. Einmal ließ ich einen Kürbis freihängen, also sein eigenes Gewicht tragen, hüllte ihn aber in wasserdichtes Guttaperchapapier und außerdem in lichtundurchlässiges schwarzes Papier ein, so daß er also in seiner Transpiration und Assimilation gehindert war. Außerdem mußte ich diesen Kürbis noch durch eine Pappschachtel bedecken, um ihn vor den heißen Sonnen- 
strahlen zu schützen, da das Guttapercha sonst teilweise weich geworden wäre. Dieses Experiment führte jedoch zu keinem Ergebnis, da sich das Einhüllen in Guttaperchapapier als ein zu schwerer Eingriff in das Leben der Pflanze erwies. Der am 2. September angesetzte Versuch mußte am 17. September beendet werden, da die Frucht stark verkümmert war - sie hatte ein gelbliches Aussehen und besaß eine runzliche Oberfläche - und der Stiel bei der Berührung abbrach. Daß die verhinderte Assimilation hierbei nicht in Betracht kommt, geht besonders aus dem gleich zu erwähnenden Sachs'schen Versuch hervor, wobei ein ganzer Zweig mit Früchten etioliert wurde, die Frucht aber trotzdem kräftig gedieh. Die unterdrückte Atmung, an die man auch denken könnte, kommt jedoch nicht in Betracht, da Guttapercha für Sauerstoff und Kohlensäure durchlässig ist. Für das Verkümmern des eingehüllten Kürbis kann also nur die unterdrückte Transpiration verantwortlich gemacht werden. Wenn aber die Transpiration einen so erheblichen Einfluß auf die Ausbildung der Frucht hat, so ist es leicht verständlich, daß eine mehr oder weniger beträchtliche Hemmung derselben, wie sie im Vöchting'schen Versuche den an der Erde liegenden Kürbis trifft, einen Einfluß auf die Gewebeausbildung hat. Wissen wir $\mathrm{ja}, \mathrm{da} \beta$ auch sonst verminderte Transpiration die Bildung der Gefäße und verholzten Elemente hemmt. Ich erinnere nur an das oben erwähnte Beispiel von Festuca ovina var. glauca ${ }^{1}$ ), wo auch das in der feuchten Atmosphäre unter der Glasglocke kultivierte Exemplar, das in seiner Transpiration stark gehindert war, keine oder fast keine Sklerenchymfasern ausbildete. Ein anderer Versuch, der den Einfluß der Ernährungsverhältnisse in anderer Weise dartun sollte, ging leider durch die Ungunst der Witterungsverhältnisse zugrunde. Ich wollte die ganze Sproßachse, an der sich der Kürbis befand, unter schlechte Ernährungsbedingungen bringen. Ich benutzte dazu eine Methode, wie sie Sachs²) $\mathrm{zu}$ einem anderen $\mathrm{Zwecke}$ anwandte. Er leitete den Gipfel eines Zweiges durch ein enges Loch in einen allseitig lichtdicht geschlossenen Kasten. Er wollte dabei demonstrieren, daß das Licht zum Wachstum nicht direkt, sondern nur wegen der durch dasselbe vermittelten Assimilation nötig ist. Die in dem Kasten belassenen Zweige entwickelten sich fast normal, da sie ihre Nährstoffe von den Blättern außerhalb des Kastens bekamen. Auch Früchte konnte er erzielen, nachdem die Blüten vorher künstlich

1) Goebel, Einleitung in die experimentelle Morphologie der Pflanzen. Leipzig u. Berlin 1908, pag. 28.

2) Sachs, Julius, Vorlesungen über Pflanzenphysiologie. Leipzig 1882, pag. $42 \pi$.

Flora, Bd. 101. 
befruchtet worden waren. Eine Hemmung zeigte sich jedoch z. B. in der Flächenentwicklung der Blätter, die nur $2 / 3-3 / 4$ der normalen betrug. Es mußte deshalb interessant erscheinen, einmal zu prüfen, ob vielleicht die histologische Ausbildung Hemmungen erfahren würde, besonders die des Fruchtstieles. Leider blieb, wie schon erwähnt, der Versuch ohne Erfolg. Da die Experimente an Cucurbito Pepo wegen Mangel an Material nicht weiter ausgedehnt werden konnten, machte ich Versuche mit anderen Cucurbitaceen, die in den Gewächshäusern des Münchener botanischen Gartens kultiviert wurden, mit Benincasa cerifera, Momordica fragrans und Luffa aegyptica. Mit letzterer machte ich nur den schon bei Cucurbita Pepo erwähnten und auch bei Benincasa und Momordica ausgeführten Versuch, die Einhüllung in schwarzes Papier und Guttaperchapapier. Doch gingen diese Versuche alle zugrunde; die Früchte entwickelten sich in der Hülle nicht weiter, wurden gelb und schrumpften, der Stiel brach dann zuletzt beim Berühren ab. Es beweist mir dies eben, daß der Ausfall des Versuches bei Cucurbita Pepo kein Zufall war und daß die Transpiration für die Ausbildung der Früchte von erheblicher Bedeutung ist. Es wurden dann einige Versuche zur. Beantwortung der Frage gemacht, ob ein an die Frucht gehängtes Gewicht, eine stärkere Ausbildung des Sklerenchyms hervorrufen könne. Ich wandte Gewichte von $1-2 \mathrm{~kg}$ an. Der Erfolg war natürlich, wie nach allem vorhergehenden zu erwarten ist, sowohl bei Momordica wie bei Benincasa ein negativer. Dann machte ich einige Versuche im Sinne Vöchting's.' Zwar hängen bei Benincasa und Momordica normal alle Früchte frei in der Luft. Doch durch Unterstützung konnte auch hier des Einfluß des Eigengewichtes ausgeschaltet werden. Der Gegensatz von an der Erde liegenden und an der Transpiration gehemmten Früchten einerseits, und frei in der Luft befindlichen andererseits wie bei Cucurbita Pepo war also hier nicht vorhanden. Es mußten demnach, wenn unsere Theorie richtig war, keine Unterschiede zu verzeichnen sein, gleichgültig, ob ich die Früchte unterstützte oder nicht. Die Versuche mit Momordica fragrans ergaben nun folgendes. Am 1. Oktober wurden die ausgewachsenen Früchte abgenommen und zwar drei Stück: Eine unterstützt und zwei freihängend kultivierte; von den zwei letzteren war eine aus unbekannten Ursachen chlorotisch geblieben. Ich gebe zunächst die Zahlen für die Gewichte dieser Früchte, sowie die Längen ihrer Stiele:

\begin{tabular}{|c|c|c|}
\hline freihängend (normal) & Gewicht der Frucht & Länge des Stieles \\
\hline freihängend (normal) : & . . $113,5 \mathrm{~g}$ & $11,5 \mathrm{~cm}$ \\
\hline freihängend (chlorotisch) & $170,5 \mathrm{~g}$ & $12 \mathrm{~cm}$ \\
\hline unterstützt & $165 \mathrm{~g}$ & $\mathrm{~cm}$ \\
\hline
\end{tabular}


Es ergab sich aus diesen Versuchen ganz eindeutig und in Übereinstimmung mit denen von Cucurbita Pepo, daß die Pflanze, gleichgültig ob sie das Gewicht ihrer Frucht zu tragen hatte oder nicht, ihren Fruchtstiel gleich ausbildet. Ja in dem Falle der unterstützten Frucht war sogar mehr Sklerenchym ausgebildet. Es liegen also individuelle Schwankungen vor, die natürlich unabhängig von mechanischer Inanspruchnahme sind. Interessant ist es auch, daß zwei der untersuchten Früchte aus einem unbekannten Grunde chlorotisch waren, also an der Assimilation verhindert waren. Trotzdem war keine Reduktion des Sklerenchyms zu beobachten. Es folgt daraus, was ja schon von vornherein sehr wahrscheinlich war, daß in diesem Falle die Assimilation wenigstens der Frucht von untergeordneter Bedeutung für

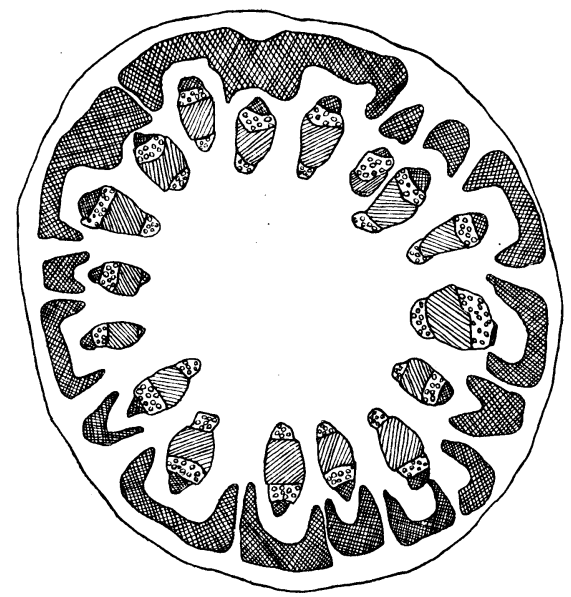

Fig. 20.

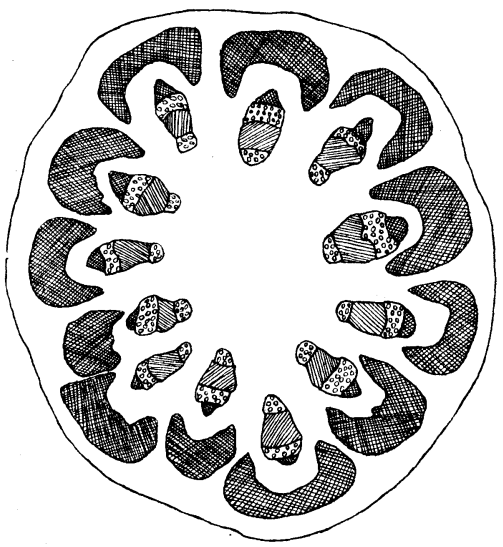

Fig. 21.

Fig. 20. Querschnitt durch den Stiel der im Kalthaus gewachsenen Frucht von Benincasa cerifera (schematisch). $=$ Sklerenchym, ${ }^{\prime \prime} /{ }^{\prime}=$ Gefäßteil, $\circ \circ=$ Siebteil der Leitbündel.

Fig. 21. Querschnitt durch den Stiel der im Victoria regia-Haus gewachsenen Frucht von Benincasa cerifera (schematisch). 嘫 $=$ Gefäßteil, $\circ$ o = Siebteil der Leitbündel.

die Bildung des mechanischen Gewebes ist. Für die große Bedeutung (der Transpiration, für die schon die Experimente der Einhüllung mit Guttaperchapapier beweisend sind, sprach auch noch eine Beobachtung an Benincasa cerifera. Betreffs des Einflusses der Unterstützung gelangte ich zu denselben Resultaten wie bei den übrigen Pflanzen, d. h. die mechanische Inanspruchnahme isi் gleichgültig für die Ausbildung von Sklerenchym. Ich verglich aber zwei Früchte miteinander, von denen die eine im Kalthaus, die andere im Victoria regia-Haus des Botanischen Gartens gewachsen war. Wie die beiden Abbildungen 
(Fig. 20 u. 21) zeigen, hat die Frucht im Kalthaus, obgleich sie nicht wesentlich schwerer war (vgl. Tabelle), erheblich mehr Sklerenchym, sowie mehr Gefäße gebildet als die im Victoria regia-Haus.

Gewicht der Frucht . . . . $1147 \mathrm{~g}$

Gewicht des Stieles . . . . $4,5 \mathrm{~g}$

Länge des Stieles . . . . . $8,5 \mathrm{~cm}$
Aus dem Kalthaus

$1960 \mathrm{~g}$

$6 \mathrm{~g}$

$6,5 \mathrm{~cm}$

Bedenkt man, daß die Luft des Victoria regia-Hauses außer seiner Wärme sich durch einen hohen Feuchtigkeitsgehalt auszeichnet, was beim Kalthaus nicht der Fall ist, so ist es verständlich, daß die durch den hohen Feuchtigkeitsgehalt herabgesetzte Transpiration in erster Linie die geringere Ausbildung des Sklerenchyms und der Gefäße bedingt hat.

Am Schlusse meiner Untersuchungen angelangt, möchte ich im folgenden eine Zusammenstellung der Resultate geben. Als allgemeinstes Ergebnis kann man wohl die Tatsache betrachten, daß die Ernährungsverhältnisse, deren großen Einfluß auf die Entwicklung und Ausbildung der äußeren Gestaltung der Pflanze von Goebel in so ausgiebiger Weise gezeigt worden ist, auch für die Histologie der Pflanze von großer Bedeutung sind.

\section{Zusammenfassung der Resultate.}

1. Die von Tschirch behauptete Differenzierung der Dikotylenwurzel in Ernährungs- und Befestigungswurzeln, die sog. Heterorhizie, ist eine nur scheinbare und beruht auf Altersunterschieden. Es gibt Übergänge zwischen beiden Typen, sowohl an der Basis verschiedener Wurzeln, als auch in verschiedener Entfernung von der Basis ein und derselben Wurzel.

2. Durch ungünstige Ernährungsbedingungen (Dunkelkultur, Abschneiden der Blätter, Abschneiden der Kotyledonen) konnte eine Reduktion der Zahl der Gefäße und der Sklerenchymfasern in den Wurzeln erreicht werden.

3. Durch dieselben Faktoren konnte bei Vicia Faba auch eine Reduktion der Zahl der Gefäßstrahlen in den Wurzeln konstatiert werden.

4. Ebenso bei Vicia Faba und Phaseolus eine Reduktion der Markzellen in der Mitte des Zentralzylinders der Wurzel bis zu deren völligem Verschwinden. Diese Hemmungserscheinung wurde bei Eintritt günstigerer Ernährungsbedingungen wieder rückgängig gemacht durch Ausbildung von Markzellen. 
Untersuchungen über die Abhängigkeit der Gefäß- u. Sklerenchymbildung usw. 219

5. Die von Wildt behauptete Anpassung an den Zug bei Wurzeln muß als Hemmungserscheinung aufgefaßt werden, weil

a) der anatomische Bau der gezogenen Wurzel gar keine zugfestere Konstruktion darstellt, als der der normalen;

b) die Zahl der Gefäße und Sklerenchymfasern und die Dicke, der ganzen Wurzel sowohl als auch des Zentralzylinders, in der gezogenen Zone rascher abnimmt als normal;

c) die Reduktion der Markzellen durch 4. als eine typische Hemmungsbildung nachgewiesen ist;

d) die Zugfestigkeit der gezogenen Strecke nicht zugenommen hat, sondern sogar etwas abgenommen.

6. Die von Vöchting vertretene Anschauung, daß eine Pflanze, die ein Organ zu tragen hat, mit dem sie für gewöhnlich nicht belastet ist, zu einer Mehrbildung von Sklerenchym veranlaßt wird, ist nicht zutreffend. Die Mehrbildung von Sklerenchym bei dem freihängend kultivierten Kürbis in dem Vöchting'schen Versuch beruht auf den günstigeren Ernährungsverhältnissen, vor allem der stärkeren Transpiration.

7. An die Pflanze angehängte Gewichte haben, wie auch schon bekannt, keinen Einfluß im Sinne einer Selbstregulation.

Ich möchte diese Arbeit jedoch nicht schließen, ohne Herrn Geheimrat von Goebel, unter dessen Leitung sie ausgeführt wurde, für seine Anregung und Unterstützung meinen besten Dank auszusprechen. 